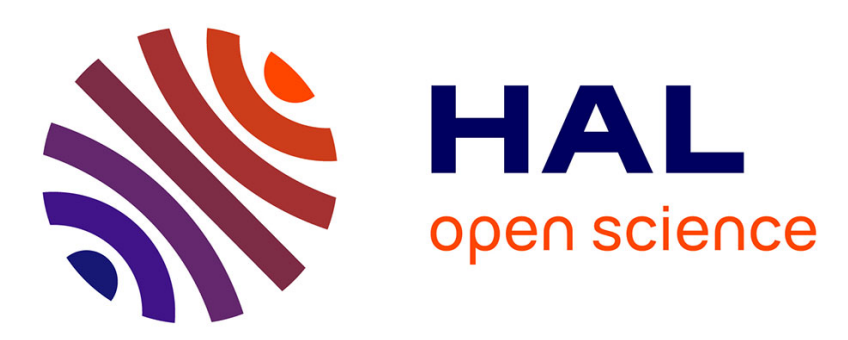

\title{
An output feedback LPV control strategy of a nonlinear electrostatic microgripper through a singular implicit modeling.
}

Mokrane Boudaoud, Marcelo Gaudenzi de Faria, Yann Gorrec, Yassine Haddab, Philippe Lutz

\section{To cite this version:}

Mokrane Boudaoud, Marcelo Gaudenzi de Faria, Yann Gorrec, Yassine Haddab, Philippe Lutz. An output feedback LPV control strategy of a nonlinear electrostatic microgripper through a singular implicit modeling.. Control Engineering Practice, 2014, 28, pp.97-111. hal-01324675

\section{HAL Id: hal-01324675 \\ https://hal.science/hal-01324675}

Submitted on 1 Jun 2016

HAL is a multi-disciplinary open access archive for the deposit and dissemination of scientific research documents, whether they are published or not. The documents may come from teaching and research institutions in France or abroad, or from public or private research centers.
L'archive ouverte pluridisciplinaire HAL, est destinée au dépôt et à la diffusion de documents scientifiques de niveau recherche, publiés ou non, émanant des établissements d'enseignement et de recherche français ou étrangers, des laboratoires publics ou privés. 


\title{
An Output feedback LPV control strategy of a nonlinear electrostatic microgripper through a singular implicit modeling
}

\author{
Mokrane Boudaoud ${ }^{*, * *}$ Marcelo Gaudenzi De Faria* \\ Yann Le Gorrec* Yassine Haddab* Philippe Lutz* \\ * FEMTO-ST Institute, UMR CNRS 6174 - UFC-ENSMM-UTBM; Automatic \\ Control and Micro-Mechatronic Systems (AS2M) Department; 24, rue Alain \\ Savary, 25000 Besançon - France \\ ** Institut des Systèmes Intelligents et de Robotique, Université Pierre et Marie \\ Curie, CNRS UMR 7222, 4 Place Jussieu, F-75252 Paris Cedex, France
}

\begin{abstract}
:
The aim of the paper is the design and the analysis of a gain scheduled controller for an accurate and fast positioning with nanometer resolution of a nonlinear electrostatic microgripper. The controller is designed to achieve a positioning of the gripping arm from few hundred nanometers to several tens of micrometers with some performance criteria. This very large operating range is crucial for a range of microrobotics applications and has never been addressed in existing control techniques of microgrippers. The controller is designed considering noises that are relevant at the nanometer scale and nonlinearities that become significant at the micrometer scale. Therefore, a nonlinear model of the system is proposed and is reformulated into a polynomial LPV (Linear Parameter Varying) model. The most relevant source of noise to be considered for the controller synthesis is defined taking into account results from previous works. Considering the particular polynomial parametric dependence of the LPV model, a multivariable controller is designed using an affine LPV descriptor representation of the system and specific linear matrix inequalities. The efficiency of the controller and the relevance of the theoretical approach are demonstrated through experimental implementation results.
\end{abstract}

Keywords: Microelectromechanical systems, Microrobotics, Linear Parameter Varying (LPV), Singular implicit modeling, $\mathrm{H} \infty$ control, Linear Matrix Inequality (LMI).

\section{INTRODUCTION}

The manipulation of micrometer sized and nanometer sized objects is very challenging in microrobotics (Chaillet et al. [2010]). For instance, single living cells manipulation has a great interest for a range of biomedical disciplines (Sun et al. [2002];Kimuraet al. [1995]). The size of biological cells is ranging from few nanometers to several tens of micrometers. Microgrippers are therefore an interesting solution to perform manipulation tasks at such scales.

MEMS (Micro Electro Mechanical systems) based microgrippers using electrostatic comb drive actuators are one of the most popular micromanipulation systems in biological research (Muntwyler et al. [2010]; Yamahata et al. [2006]). Although, comb drive actuators are highly nonlinear when exceeding few micrometer displacements (Legtenberg et al. [1996]), they allow positioning gripping arms over several tens of micrometers, they have no hysteretic behavior and they do not generate heating.

Nonlinearities of comb drive actuators are due to electrostatic potentials (Lee et al. [2007]) and axial forces acting on compliant mechanisms (Legtenberg et al. [1996]). The side instability is also a non-linearity that occurs when the gap spacing between fixed and movable electrodes of the actuator is no longer constant (Alwi et al. [2012]). It is therefore very difficult to control such actuators with

* E-mail: mokrane.boudaoud@isir.upmc.fr;marcelo.gaudenzi@femtost.fr;legorrec@femto-st.fr;yassine.haddab@femto-st.fr;

philippe.lutz@femto-st.fr robust performances in a wide operating range. This is nevertheless fundamental for an efficient manipulation of fragile samples such as biological cells regardless their size.

An extensive literature is available for the control of comb drive actuators (Bryzek et al. [2003];Borovic et al. [2006]). Open loop control strategies are very useful for MEMS (Acar et al. [2001]) but they are most of time not robust w.r.t model uncertainties and nonlinear dynamics. Linear closed loop controllers are often sufficient to ensure the stability and robust performances when the actuator is driven for few micrometers only. The linear quadratic Gaussian control (Boudaoud et al. [2013]) is powerful to filter dynamic noises but requires a full state estimate and is not robust w.r.t model uncertainties and environmental disturbances. Linear time invariant $\mathrm{H} \infty$ control (Haddab et al. [2008]) can improve both positioning precision and bandwidth of the actuator but is limited to satisfy robust performances in a wide operating range due to fixed weights. The $\mathrm{H} \infty$ loop shaping controller is useful to control multiple degrees of freedom of MEMS by decoupling several input/output transfers functions and satisfying robust performances (Pang et al. [2009]). Direct and indirect sliding mode control with an adaptive gain (Fei et al. [2009]) or with an observer (Fei et al. [2007]) proved its efficiency to control comb drive actuators in MEMS gyroscopes. Closed loop stability and robust performances are satisfied with an on-line identification of the upper bounds of uncertainties (Fei et al. [2009]) or by considering a single non-linearity such as the damping (Ebrahimi et al. [2006]) for the controller synthesis. To deal with the side 
instability, linear and nonlinear control approaches have been proposed. They are based on input/output linearization (Maithripala et al. [2003]), differential flatness (Zhu et al. [2005]), backstepping (Salah et al. [2010]) or robust PID (Vagia et al. [2008]). Recent works demonstrated the interest of Linear Parameter Varying (LPV) controllers to deal with this non-linearity (Shirazi et al. [2011]; Alwi et al. [2012]). Such controllers allow driving the actuator beyond the side instability (generally less than $10 \mu \mathrm{m}$ displacement). Nevertheless, they do not provide degrees of robustness to matched nonlinearities such as damping and stiffness variation. The problem of positioning comb drive actuators from few hundred nanometers to several tens of micrometers is not addressed. Moreover, the design of the controllers assumes that the MEMS is working in ideal conditions and the effect of environmental noises on the positioning resolution is not considered. Such issues have not been analyzed previously and remain an open research area although it is crucial for MEMS based microgrippers.

The aim of this paper is to provide a new approach to control MEMS based microgrippers using comb drive actuators. A multivariable controller is designed through the LPV theory in order to ensure the stability and some closed loop performances (precision, nanometer resolution, no overshoot, fast response time) over a very wide operating range despite significant noises and nonlinearities. Nonlinearities arise from a large variation of the stiffness and the damping when increasing the displacement of the actuator. Nonlinear parameters, namely the stiffness and the damping, are matched. Therefore instead of using them "independently" as scheduling variables, the use of only one scheduling parameter is proposed. To do so, a multivariable polynomial LPV model of the system is introduced. The polynomial parametric dependence of the model causes some issues for the derivation of the controller. As such, an affine LPV descriptor model and a finite number of linear matrix inequalities are used for the controller synthesis. In order to achieve resolution requirement, the main source of noise to be considered for the controller synthesis is defined taking into account results from previous works (Boudaoud et al. [2011]; Boudaoud et al. [2012]). A specific weighting function is introduced to ensure a high positioning resolution.

The relevance of the control approach is demonstrated considering a commercial microgripper (FT-G100 from FemtoTools Company) and shows that large positioning with nanometer resolution can be achieved using two sensors only and does not require the use of expensive vibration isolation tables commonly employed in microrobotics which is a great advantage from application point of view. The paper is organized as follow. In section 2 , the global architecture of the microgripper is presented. Sections 3 and 4 deal with the nonlinear modeling and the LPV modeling of the actuation mechanism. The proposed control strategy is presented in the fifth section. In section 6 , experimental control results are presented.

\section{FEATURES OF THE MICROGRIPPER}

The FT-G100 microgripper is designed to handle objects ranging from $1 \mu \mathrm{m}$ to $100 \mu \mathrm{m}$. To pick up an object, an actuated arm is pushed toward closure thanks to a comb drive actuator. The base of the actuated arm is fixed to a flexure joint Fig.1 and a suspension mechanism including two pairs of clamped-clamped beams holds the movable part of the actuator. While the gripping arms are closed around an object, the deflection of the sensing arm is detected by a capacitive sensor. The sensor consists of a transverse comb-drive with a differential capacity proportional to the displacement of the movable fingers. This displacement (due to the gripping force) is translated into analog voltage $V_{\text {out }}$ throughout a MS3110 readout chip (Irvine Sensors). Two pairs of clamped-clamped beams and a flexure joint are also attached to the sensor and the base of the sensing arm respectively.

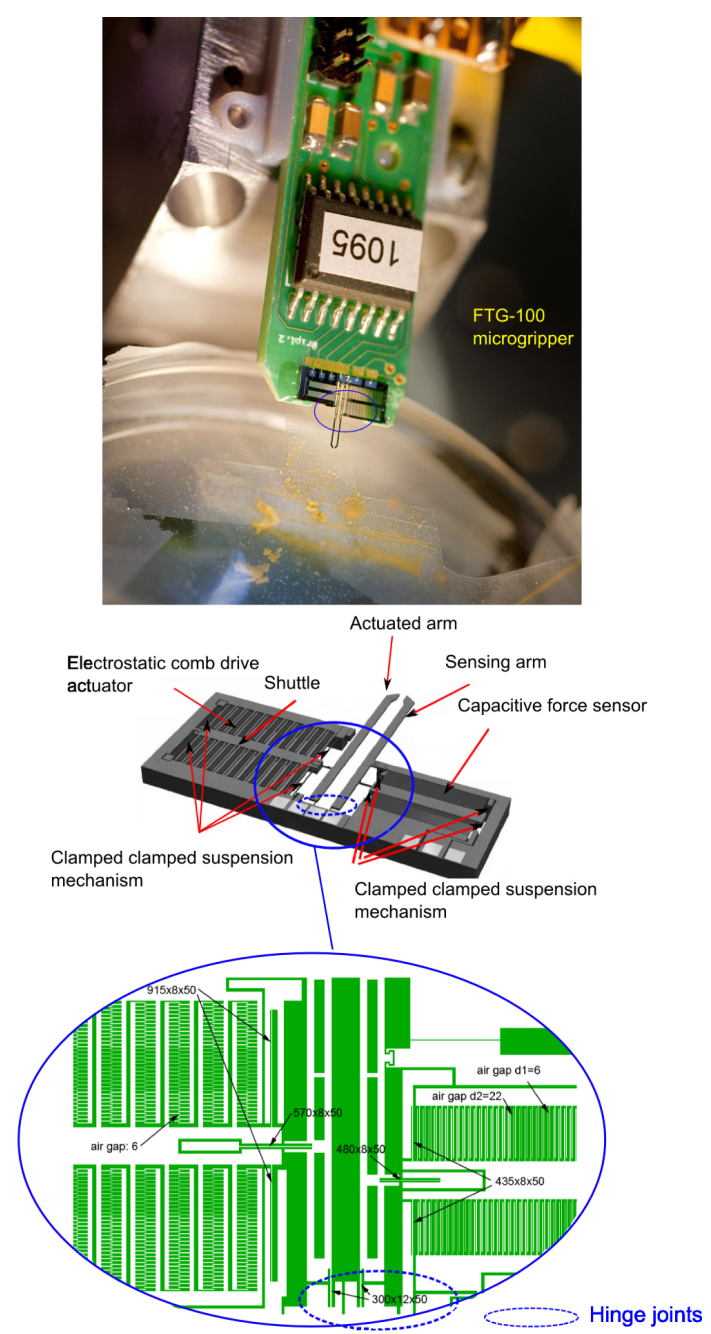

Fig. 1. Structure of the FT-G100 microgripper (FemtoTools $\mathrm{GmbH})$.

Table 1. Dimensions of the actuation mechanism

\begin{tabular}{lll}
\hline & Actuated arm & Doubly-clamped beams \\
\hline Length & $L=5150 \mu \mathrm{m}$ & $L_{s}=915 \mu \mathrm{m}$ (half length) \\
\hline Width & $l=150 \mu \mathrm{m}$ & $l_{s}=50 \mu \mathrm{m}$ \\
\hline Thickness & $e=50 \mu \mathrm{m}$ & $e_{s}=8 \mu \mathrm{m}$ \\
\hline
\end{tabular}

The FT-G100 microgripper is used in many robotics laboratories. Very few control solutions are nevertheless provided by the manufacturer. In this work, the designed controller can be applied to any MEMS based microgripper using a comb drive actuator (Yamahata et al. [2006];Beyeler et al. [2007];Muntwyler et al. [2010];Vijayasai et al. [2010]) and leads to new perspectives for high precision micro/nano-manipulation tasks.

\section{NONLINEAR MODELING AND IDENTIFICATION}

The aim is the accurate position control of the actuated $\operatorname{arm} \operatorname{tip} y_{a}(L)$ in the direction of $\mathrm{y}$ axis (see Fig.2). In this section, a nonlinear dynamic model of the actuation mechanism is proposed. The model describes the dynamic transfer between the input voltage $V_{i n}$ and the position 
$y_{a}(L)$. Input noises are also considered. Static and dynamic parameters of the model are identified experimentally and a final nonlinear model is derived for the control.

\subsection{Nonlinear dynamic modeling}

The modeling approach is performed under the following assumptions (see Boudaoud et al. [2013] for assumptions verification): (i) the actuated arm is rigid in the direction of y axis, (ii) the slender beam between the shuttle and the actuated arm is not subject to buckling and (iii) the pseudo rigid beams behaves as a hinge joint.
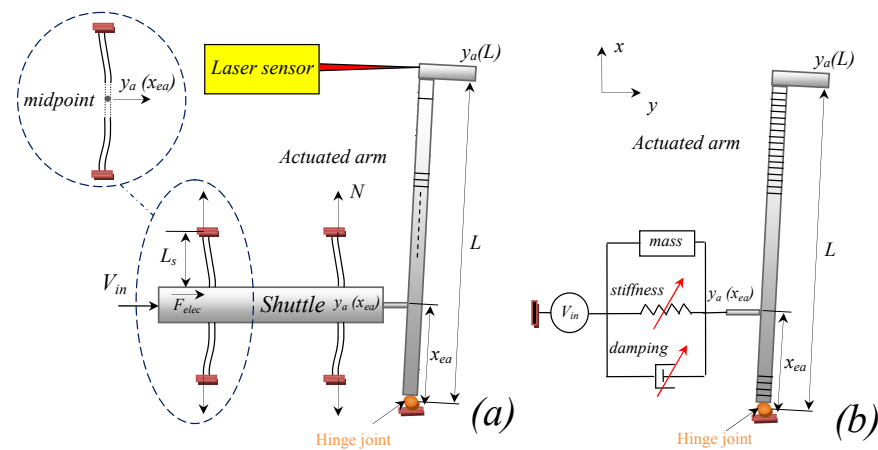

Fig. 2. Simplified scheme of the actuation mechanism (a) and equivalent scheme of suspensions (b).

Therefore, let us consider a nonlinear Euler Bernoulli equation describing the undamped bending of the suspension mechanism at the midpoint of the doubly clamped beams $y_{a}\left(x_{e a}\right)$ in the direction of y axis (see Fig.2.a):

$$
\begin{aligned}
E I_{s} \frac{\partial^{4} y_{a}\left(x_{e a}\right)}{\partial x^{4}} & +\rho l_{s} e_{s} \frac{\partial^{2} y_{a}\left(x_{e a}\right)}{\partial t^{2}} \\
& +N \frac{\partial^{2} y_{a}\left(x_{e a}\right)}{\partial x^{2}}=F_{\text {elec }} \delta\left(x-L_{s}\right)
\end{aligned}
$$

where $E$ is the Young's modulus, $I_{s}$ is the area moment of inertia of the doubly-clamped beams, $\rho$ is the mass density, $F_{\text {elec }}$ is the electrostatic force and $\delta$ is the Dirac delta function. Moreover, $L_{s}, l_{s}$ and $e_{s}$ are dimensions as described in Table.1.

The two first terms of equation (1) relates to the linear Euler Bernoulli beam equation. The third term introduces a non-linearity due to the so called inplane tension located at the clamped parts of the suspensions (see Fig.2.a). The inplane tension $N$ is given by (Kovacicv et al. [2011]):

$$
N=\frac{E l_{s} e_{s}}{4 L_{s}} \int_{0}^{2 L_{s}}\left(\frac{\partial y_{a}}{\partial x}\right)^{2} \partial x
$$

The electrostatic force is governed by the following equation (Moussa et al. [2002]):

$$
F_{\text {elec }}=\frac{N_{a} \varepsilon h_{z}}{2 g} \cdot V_{\text {in }}^{2}
$$

where $V_{i n}$ is the actuation voltage, $N_{a}=1300$ is the total number of fingers (movable and fixed) in the actuator, $\varepsilon=8.85 \mathrm{pF} / \mathrm{m}$ is the permittivity of the dielectric material (air), $h_{z}=50 \mu \mathrm{m}$ is the thickness of comb fingers, and $g=6 \mu m$ is the gap spacing between two fingers.

Considering the assumptions of the modeling, the variable $y_{a}(L)$ is introduced in equation (1) leading to:

$$
\begin{aligned}
\frac{E I_{s}}{D_{a}} \frac{\partial^{4} y_{a}(L)}{\partial x^{4}} & +\frac{\rho l_{s} e_{s}}{D_{a}} \frac{\partial^{2} y_{a}(L)}{\partial t^{2}} \\
& +\frac{N}{D_{a}} \frac{\partial^{2} y_{a}(L)}{\partial x^{2}}=F_{\text {elec }} \delta\left(x-L_{s}\right)
\end{aligned}
$$

where $D_{a}=L / x_{e a}$ is an amplification parameter $\left(x_{e a}=\right.$ $1100 \mu \mathrm{m})$.

Substituting equation (2) into (4) gives the partial differential equation:

$$
\begin{aligned}
& \frac{E I_{s}}{D_{a}} \frac{\partial^{4} y_{a}(L)}{\partial x^{4}}+\frac{\rho l_{s} e_{s}}{D_{a}} \frac{\partial^{2} y_{a}(L)}{\partial t^{2}} \\
& +\left[\frac{E l_{s} e_{s}}{4 L_{s} D_{a}} \int_{0}^{2 L_{s}}\left(\frac{\partial y_{a}}{\partial x}\right)^{2} \partial x\right] \frac{\partial^{2} y_{a}(L)}{\partial x^{2}}=F_{\text {elec }} \delta\left(x-L_{s}\right)
\end{aligned}
$$

Thus, using the perturbation method (see (Kovacicv et al. [2011]) for more information), equation (5) can be simplified into a lumped parameter equation of the first Eigen mode of the system as follows:

$$
m_{a_{0}} \frac{\partial^{2} y_{a}(L)}{\partial t^{2}}+k_{1_{a}} y_{a}(L)+k_{3_{a}} y_{a}^{3}(L)=\frac{1}{D_{a}} F_{\text {elec }}
$$

with:

$$
\begin{gathered}
m_{a_{0}}=\frac{\rho l_{s} e_{s}}{\phi_{1}^{2}\left(2 L_{s}\right) D_{a}^{2}} \int_{0}^{2 L_{s}} \phi_{1}^{2}(x) . \partial x \\
k_{1 a}=\frac{2 E I_{s}}{\phi_{1}^{2}\left(2 L_{s}\right) D_{a}^{2}} \int_{0}^{2 L_{s}}\left(\phi_{1}^{\prime \prime}(x)\right)^{2} . \partial x \\
k_{3 a}=\frac{E}{\phi_{1}^{2}\left(2 L_{s}\right) D_{a}^{2}}\left(\int_{0}^{2 L_{s}}\left(\phi_{1}^{\prime}(x)\right)^{2} . \partial x\right)^{2}
\end{gathered}
$$

The parameter $\phi_{1}$ is the shape mode of the first Eigen mode of the suspensions. $k_{1 a}$ is the effective linear stiffness of the actuation mechanism. The nonlinear term $k_{3 a}$ is the cubic stiffness (it is due to the inplane tension $N$ ).

In the partial differential equation (1) and in equation (6) , the damping of the system is not taking into account and only the mass of the suspension mechanism (i.e. doubly clamped beams) is considered.

Hence, in order to account the mass of the complete actuation mechanism, the mass $M_{s h}$ is added to $m_{a_{0}}$. The parameter $M_{s h}$ is the mass of the shuttle and that of the actuated arm. Moreover, to account the real dynamic behavior of the system, the term $d_{a}$ is introduced to define the damping of the actuation mechanism.

As such, equation (6) becomes:

$m_{a} \frac{\partial^{2} y_{a}(L)}{\partial t^{2}}+d_{a} \frac{\partial y_{a}(L)}{\partial t}+k_{1_{a}} y_{a}(L)+k_{3_{a}} y_{a}^{3}(L)=\frac{1}{D_{a}} F_{\text {elec }}$

where $m_{a}=m_{a_{0}}+M_{s h}$ is the mass of the actuation mechanism.

In the next section, both static and dynamic parameters of the nonlinear model are identified using experimental data from a commercial FT-G100 microgripper. 


\subsection{Identification}

A high resolution $(0.01 \mathrm{~nm})$ laser interferometer (SP-120 SIOS Metechnik $\mathrm{GmbH}$ ) is used to perform measurements of the displacement $y_{a}(L)$ at the tip of the actuated arm (see Fig.3). Input voltages are generated using a dSPACE control board (DS1103). First, $k_{1 a}$ and $k_{3 a}$ are identified through the experimental static characteristic $y_{a}(L) / V_{i n}$ for $0<V_{i n}<200 \mathrm{~V}$. Experimental data are then fitted using equation (7) in static mode.

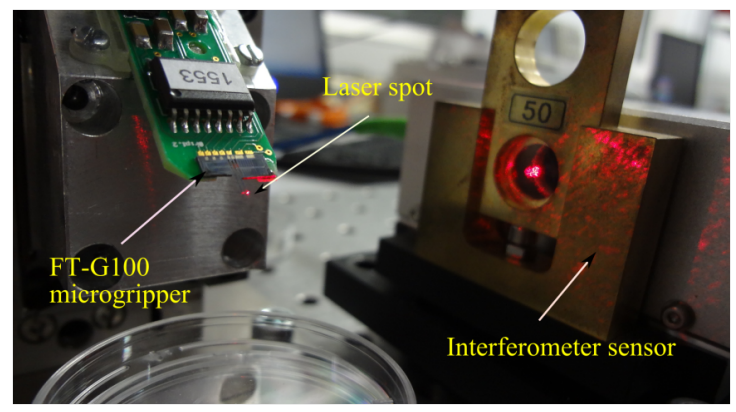

Fig. 3. Measurement of the actuated arm position using a laser interferometer sensor.

Let us recall that in the modeling part, equation (6) is related to the Euler Bernoulli equation (1). This modeling approach justify and explain why a cubic stiffness term $k_{3 a}$ appear in the lumped parameter equation (6). However, from experimental data, it has been shown that for the FT-G100 actuation mechanism, a third order polynomial (related to the nonlinear stiffness with a cubic term) is not sufficient to describe accurately the static characteristic $y_{a}(L) / V_{i n}$. The mean error of the static characteristic in the whole operating range of the system is found to be equal to $17.11 \%$. . The reason is that in addition to the inplane tension (which produce the cubic stiffness $k_{3 a}$ ), other sources of nonlinearities such electrostatic ones can produce nonlinear stiffness terms of higher orders (Ye et al. [1998]). Therefore, in this study, we increase the order of the nonlinear stiffness in order to reduce the mean fitting error to less than $10 \%$. The nonlinear stiffness is described by a sixth order polynomial leading to the following expression:

$$
k_{a}=\sum_{i=1}^{6} k_{i a} y_{a}^{i-1}(L)=\frac{1}{D_{a} y_{a}(L)} F_{\text {elec }}
$$

As such, in static mode, the equation governing the displacement $y_{a}(L)$ becomes:

$$
\begin{aligned}
& k_{1 a} y_{a}(L)+k_{2 a} y_{a}^{2}(L)+k_{3 a} y_{a}^{3}(L)+k_{4 a} y_{a}^{4}(L) \\
& +k_{5 a} y_{a}^{5}(L)+k_{6 a} y_{a}^{6}(L)=\frac{1}{D_{a}} F_{\text {elec }}
\end{aligned}
$$

Equation (9) is not deduced from equation (6) when $t \rightarrow+\infty$. Only the parameters $y_{a}(L), F_{\text {elec }}, k_{1 a}, k_{3 a}$ and $D_{a}$ are the same in the two equations. Other terms are due to the polynomial order extension.

The mean fitting error is then reduced to $3.66 \%$ and results are presented in Fig.4.a. The identified linear, quadratic, cubic and higher order stiffness terms are given in Table.2. Consequently, the nonlinear characteristic of the stiffness is deduced as shown in Fig.4.b.

Thereafter, step voltages are applied to the actuator with amplitudes ranging from $5 \mathrm{~V}$ to $200 \mathrm{~V}$ (40 steps exci-
Table 2. Identified static and dynamic parameters of the actuation mechanism.

\begin{tabular}{lll}
\hline Symbol & Value & Description \\
\hline \hline$m_{a}$ & $3.9843 \times 10^{-8} \mathrm{~kg}$ & mass \\
\hline$k_{1 a}$ & $1.85 \mathrm{~N} / \mathrm{m}$ & stiffness terms \\
$k_{2 a}$ & $-1.6 \times 10^{3} \mathrm{~N} / \mathrm{m}^{2}$ & \\
$k_{3 a}$ & $4.88 \times 10^{8} \mathrm{~N} / \mathrm{m}^{3}$ & \\
$k_{4 a}$ & $-1.69 \times 10^{12} \mathrm{~N} / \mathrm{m}^{4}$ & \\
$k_{5 a}$ & $-3.98 \times 10^{16} \mathrm{~N} / \mathrm{m}^{5}$ & \\
$k_{6 a}$ & $4.71 \times 10^{20} \mathrm{~N} / \mathrm{m}^{6}$ & \\
$d_{0 a}$ & $1.76 \times 10^{-6} \mathrm{Ns} / \mathrm{m}$ & damping terms \\
$d_{1 a}$ & $0.378 \mathrm{Ns} / \mathrm{m}^{2}$ & \\
$d_{2 a}$ & $-1.58 \times 10^{4} \mathrm{Ns} / \mathrm{m}^{3}$ & \\
$d_{3 a}$ & $1.67 \times 10^{8} \mathrm{Ns} / \mathrm{m}^{4}$ & \\
$d_{4 a}$ & $2.47 \times 10^{12} \mathrm{Ns} / \mathrm{m}^{5}$ & \\
\hline
\end{tabular}

tation are applied). For each excitation step, the response $y_{a}(L)$ is measured experimentally. The mass is identified from the step response which corresponds to $5 \mathrm{~V}$ step excitation and the damping is identified at each operating point (from $5 \mathrm{~V}$ to $200 \mathrm{~V}$ ). Results show that the damping is nonlinear. It increases with increasing the amplitude of the step excitation starting from $y_{a}(L)=60 \mu \mathrm{m}$ (see Fig.4.c).
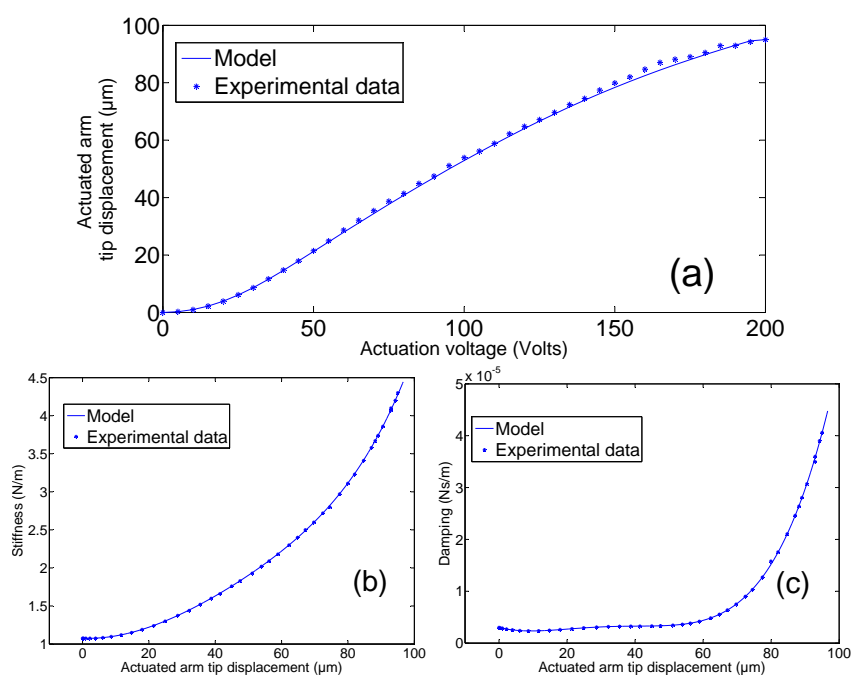

Fig. 4. Nonlinear characteristics of the FT-G100 actuation mechanism (a) $y_{a}(L) / V_{i n}$, (b) $k_{a} / V_{i n}$ and (c) $d_{a} / V_{i n}$.

To describe accurately the damping of the system, it has been sufficient to model it by a fourth order polynomial (using identification results) of the form:

$$
d_{a}=\sum_{i=0}^{4} d_{i a} y_{a}^{i}(L)
$$

Taking into account the nonlinear terms of the stiffness and the damping, the nonlinear state space model of the actuation mechanism is given as:

$$
\left\{\begin{array}{l}
{\left[\begin{array}{l}
\dot{y}_{a}(L) \\
\ddot{y}_{a}(L)
\end{array}\right]=\left[\begin{array}{c}
0 \\
-\frac{1}{m_{a}} \sum_{i=1}^{6} k_{i a} y_{a}^{i-1}(L)-\frac{1}{m_{a}} \sum_{i=0}^{4} d_{i a} y_{a}^{i}(L)
\end{array}\right]\left[\begin{array}{l}
y_{a}(L) \\
\dot{y}_{a}(L)
\end{array}\right]} \\
+\left[\begin{array}{l}
0 \\
\frac{K_{e}}{m_{a} D_{a}}
\end{array}\right] \cdot V_{i n}^{2} \\
y_{a}(L)=\left[\begin{array}{ll}
1 & 0
\end{array}\right]\left[\begin{array}{l}
y_{a}(L) \\
\dot{y}_{a}(L)
\end{array}\right] \\
K_{e}=\frac{N_{a} \varepsilon h_{z}}{2 g}
\end{array}\right.
$$


To validate the modeling approach based on nonlinear polynomial terms, frequency responses of the model are compared with experimental ones. For each operating point, experimental and simulated step responses are recorded and a Fast Fourier Transform (FFT) is computed. Results are presented in Fig.5.

The nonlinear model describes accurately the increase of the fundamental resonance frequency with increasing the input voltage due to the nonlinear stiffness. The fundamental resonance frequency increases from $827 \mathrm{~Hz}$ to more than $2 \mathrm{kHz}$ for $5 \mathrm{~V}<V_{\text {in }}<175 \mathrm{~V}$. For $V_{\text {in }}>70 \mathrm{~V}$, higher order eigenmodes can be observed experimentally. The model is able to capture this characteristic. For $V_{i n}>$ $155 \mathrm{~V}$, the value of the fundamental resonance frequency obtained by simulation does not agree with experiments. This is due to the fact that the actuated arm is close to the sensing arm. Hence, the overshoot of the step response causes a contact between the two arms. This contact is not considered in the model.
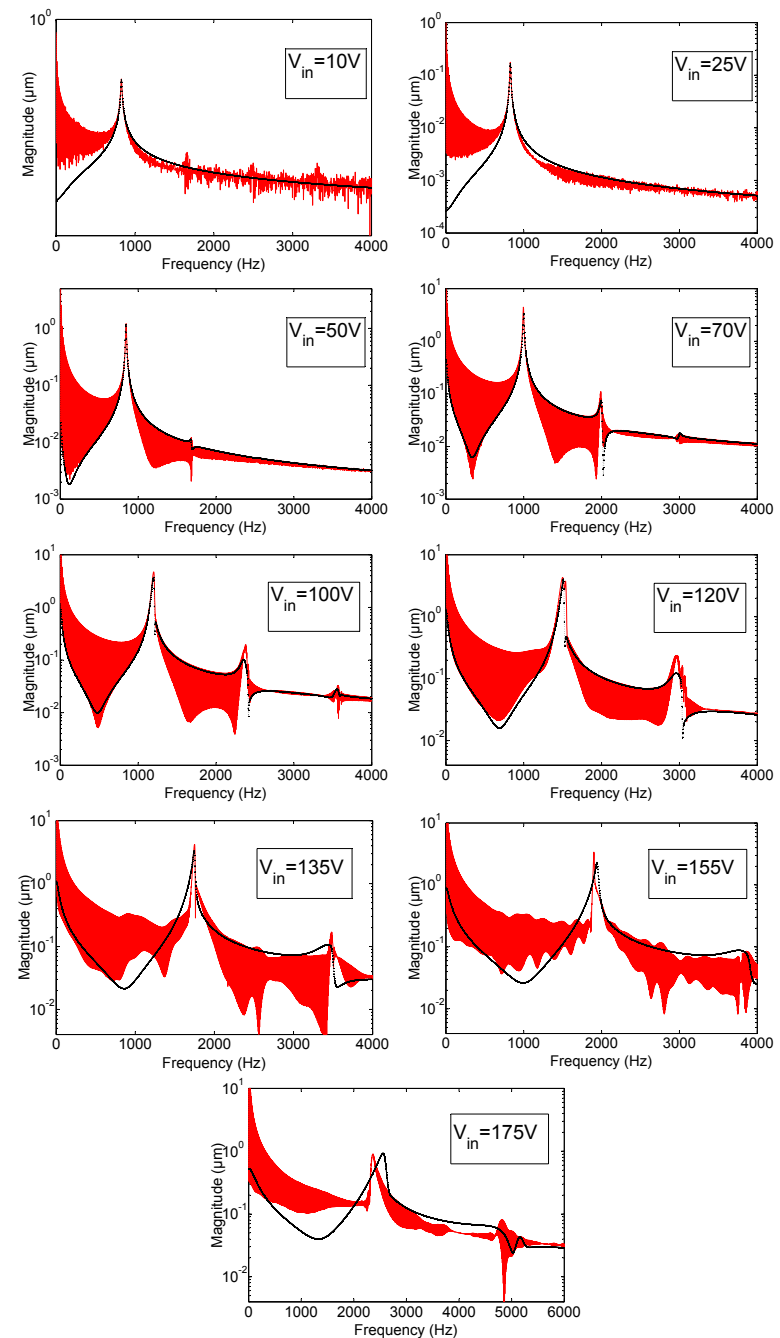

Fig. 5. Frequency responses of the nonlinear actuation mechanism: experimental data (continuous line) and simulation data (dashed line).

\subsection{Noise influences}

In (Boudaoud et al. [2011]) and (Boudaoud et al. [2012]), a detailed study on environmental noises and their effect on the positioning resolution of micromanipulation systems is performed. Three main sources of noise have been studied: cultural noises (ground vibrations), acoustic noises and thermal noises. Models and experiments allowed the determination of the frequency range and the root mean square (r.m.s) amplitude of each source of noise:

(1) The power spectrum of the cultural noise has a $\frac{10^{-11}}{f^{4}}$ shape ( $f$ is the frequency) (see Fig.6). In the frequency range $[1 \mathrm{~Hz} 100 \mathrm{~Hz}]$, the r.m.s amplitude of the cultural noise is about $223 \mathrm{~nm}$. The r.m.s amplitude reaches $6 \mathrm{~nm}$ and $1.2 \mathrm{~nm}$ in the frequency ranges $[100 \mathrm{~Hz} \quad 250 \mathrm{~Hz}]$ and $[300 \mathrm{~Hz} 450 \mathrm{~Hz}]$ respectively.

(2) Acoustic noise can amplify the r.m.s amplitude of the cultural noise by a factor of ten beyond $35 \mathrm{~dB}$ Sound Pressure Level (SPL). This threshold of acoustic noise can be easily reached in laboratories environments.

(3) The thermal noise is a pseudo white noise. Its r.m.s amplitude is related to the damping of a mechanical system. The thermal noise is often considered as the main limitation of the positioning resolution in MEMS. In (Boudaoud et al. [2012]), it has been found that the positioning resolution of AFM (Atomic Force Microscopy) based microgrippers is ranging from $365 \mathrm{pm}$ to $675 \mathrm{pm}$. It is shown that resolutions are limited by both thermal and acoustic noises.

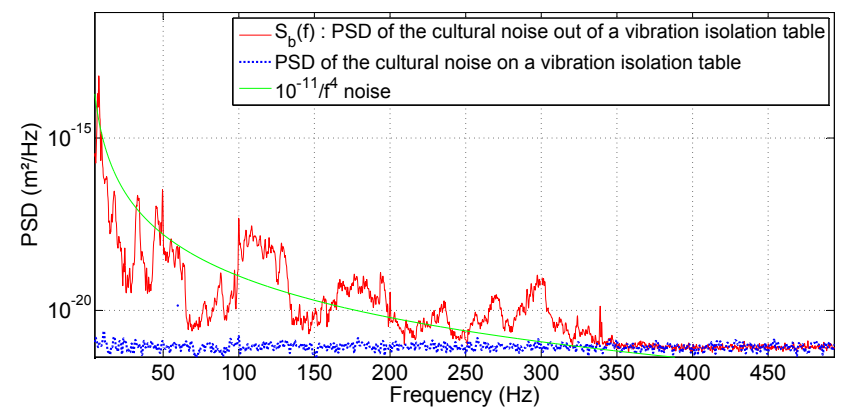

Fig. 6. PSD of the cultural noise measured on and out of a vibration isolation table (Boudaoud et al. [2011]). The cultural noise $b(t)$ considered in this paper relates to the noise measured out of the vibration isolation table. Its PDS has a $10^{-11} / f^{4}$ shape.

Both the cultural noise and the thermal noise will be considered as input signals (Fig.7) to define their effect on the positioning resolution of the actuation mechanism.

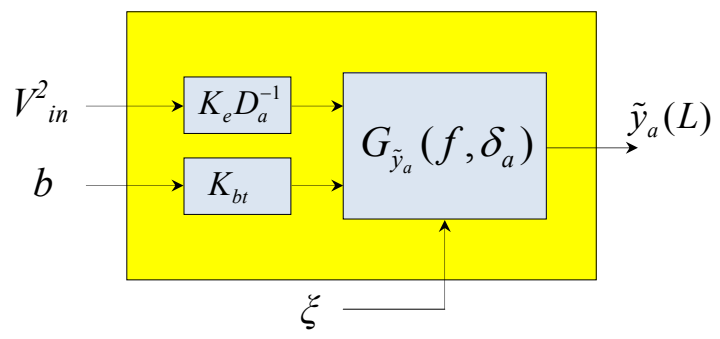

Fig. 7. The actuation mechanism subject to an input voltage $V_{i n}$, an input $10^{-11} / f^{4}$ noise (cultural noise b) and an input white noise (thermal noise $\xi$ ).

\section{Cultural noise}

The cultural noise $b(t)$ is introduced at the input of the nonlinear model (11) such that the dynamic transfer $G_{y_{a}(L)_{/ b}}$ satisfies (Younis et al. [2011]):

$$
\left\{\begin{array}{l}
\lim _{f \rightarrow 0} G_{y_{a}(L)_{/ b}}(f)=1 \\
G_{y_{a}(L)_{/ b}}\left(f=f_{r a}\right)=Q_{a}=d_{a}^{-1} \sqrt{m_{a} k_{a}}
\end{array}\right.
$$


where $f_{r a}$ and $Q_{a}$ are respectively the fundamental resonance frequency and the quality factor of the actuation mechanism.

These conditions allow introducing $b(t)$ in (11) as:

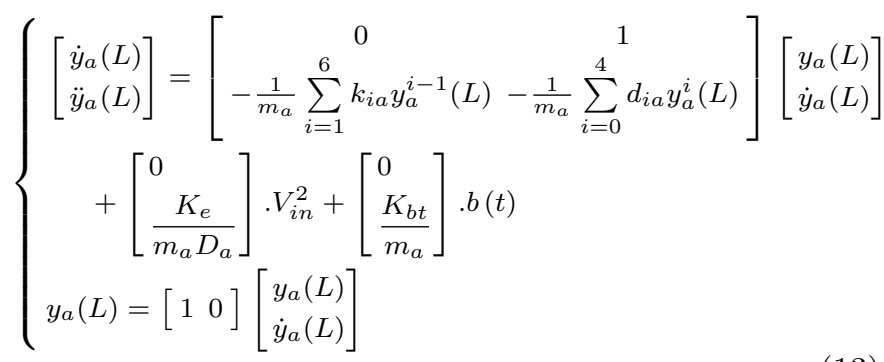

with:

$$
K_{e}=\frac{N_{a} \varepsilon h_{z}}{2 g}, \quad K_{b t}=\sum_{i=1}^{6} k_{i a} y_{a}^{i-1}(L)
$$

Therefore, around an operating point $\delta_{a}$, the Power Spectral Density (DSP) of the displacement $\tilde{y}_{a}(L)=$ $y_{a}(L)-\delta_{a}$ in response to the cultural noise can be deduced from the following relation:

$$
S_{c u l t}\left(f, \delta_{a}\right)=\left|K_{b t} G_{\tilde{y}_{a}}\left(f, \delta_{a}\right)\right|^{2} S_{b}(f)
$$

where

$$
G_{\tilde{y}_{a}}\left(f, \delta_{a}\right)=\frac{1}{m_{a} s^{2}+d_{a}\left(\delta_{a}\right) s+k_{a}\left(\delta_{a}\right)}
$$

with:

$$
k_{a}\left(\delta_{a}\right)=\sum_{i=1}^{6} i k_{i a} \delta_{a}^{i-1}, \quad d_{a}\left(\delta_{a}\right)=\sum_{i=0}^{4} d_{i a} \delta_{a}^{i}
$$

$S_{\text {cult }}\left(f, \delta_{a}\right)$ and $S_{b}(f)$ are respectively the PSD of the displacement $\tilde{y}_{a}$ (in response to the cultural noise) and the PSD of the cultural noise.

\section{Thermal noise}

The thermal noise is considered as the main source of noise that limits the absolute resolution of MEMS (Younis et al. [2011]). For this reason, this source of noise is initially considered in the study. In the case of the FTG100 actuation mechanism, one can describe the PSD of the displacement $\tilde{y}_{a}(L)=y_{a}(L)-\delta_{a}$ in response to the thermal noise as follow:

$$
S_{\text {therm }}\left(f, \delta_{a}\right)=\left|G_{\tilde{y}_{a}}\left(f, \delta_{a}\right)\right|^{2} S_{\xi}\left(f, \delta_{a}\right)
$$

where $S_{\text {therm }}\left(f, \delta_{a}\right)$ and $S_{\xi}\left(f, \delta_{a}\right)$ are respectively the PSD of the displacement $\tilde{y}_{a}$ (in response to the thermal noise) and the PSD of the thermal noise.

The thermal noise $\xi$, also called Langevin force, is a white noise. Its PSD is related to the damping of the mechanical system, the temperature $\mathrm{T}$ expressed in Kelvin and the Boltzman constant $K_{B}=1.38 \times 10^{-23} \mathrm{~J} /$ Kelvin (Callen et al. [1951]).

In the case of the actuation mechanism:

$$
S_{\xi}(f)=4 \cdot K_{B} \cdot T \cdot d_{a}\left(\delta_{a}\right)
$$

Equations (15) and (16) are then used to define the PSD of the displacement $\tilde{y}_{a}$ when the actuation mechanism is subject to the thermal noise:

$$
\begin{aligned}
& S_{\text {therm }}\left(f, \delta_{a}\right)=\frac{4 K_{B} T m_{a}}{k_{a}\left(\delta_{a}\right) d_{a}\left(\delta_{a}\right)} \\
& \times\left[\left(1-4 \pi^{2} \frac{m_{a}}{k_{a}\left(\delta_{a}\right)} f^{2}\right)^{2} \frac{m_{a} k_{a}\left(\delta_{a}\right)}{d_{a}\left(\delta_{a}\right)}+4 \pi^{2} \frac{m_{a}}{k_{a}\left(\delta_{a}\right)} f^{2}\right]^{-1}
\end{aligned}
$$

To define the effect of the cultural noise and that of the thermal noise on the resolution of the system, $S_{c u l t}\left(f, \delta_{a}\right)$ and $S_{\text {therm }}\left(f, \delta_{a}\right)$ are computed for $\delta_{a} \in[5 \mu \mathrm{m} 100 \mu \mathrm{m}]$. In order to compute $S_{\text {cult }}\left(f, \delta_{a}\right)$, the PSD of the cultural noise set at the model input is $S_{b}=\frac{10^{-11}}{f^{4}}$. The temperature is set at $22^{\circ} C$ for the computation of $S_{\text {therm }}\left(f, \delta_{a}\right)$. Results are presented in Fig.8.

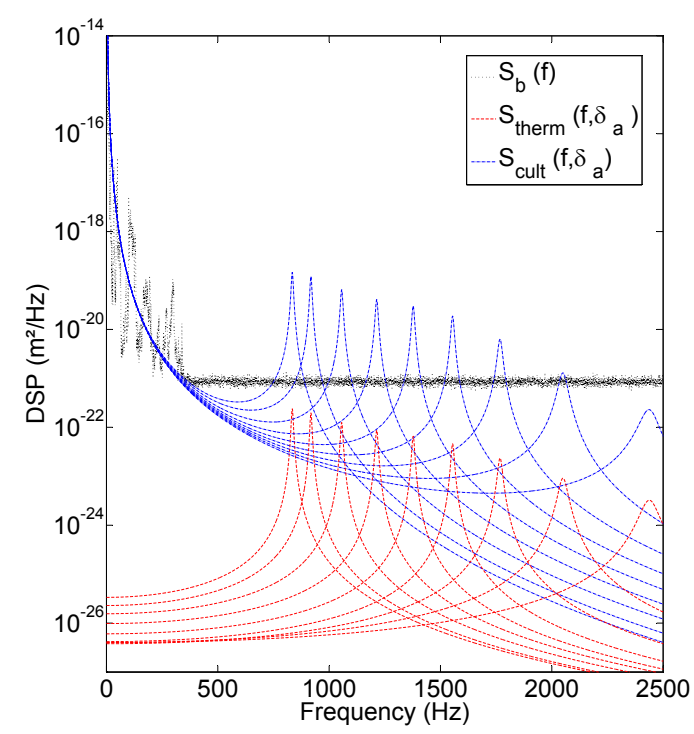

Fig. 8. PSD of the cultural noise $\left(S_{b}(f)\right)$ and PSD of $\tilde{y}_{a}(L)$ in response to the cultural noise $\left(S_{\text {cult }}\left(f, \delta_{a}\right)\right)$ and in response to the thermal noise $\left(S_{\text {therm }}\left(f, \delta_{a}\right)\right)$ for $\delta_{a} \in[5 \mu m 100 \mu m]$.

The r.m.s. values of $\tilde{y}_{a}$ are derived from the PDSs according to:

$$
r . m . s_{\tilde{y}_{a}(L)}\left(f_{1}, f_{2}\right)=\sqrt{\sum_{f_{1}}^{f_{2}} P S D(f) \Delta f}
$$

With $\Delta f=f_{2}-f_{1}=1 k H z$. The r.m.s of $\tilde{y}_{a}$ is the absolute positioning resolution.

When the system is subject to the thermal noise only, the absolute positioning resolution of the actuation mechanism is $59.8 \mathrm{pm}$. Such a displacement cannot be measured by the laser interferometer due to its resolution $(0.01 \mathrm{~nm})$. On the other hand, the absolute positioning resolution of the system subject to the cultural noise only is much higher. Results show that r.m.s. $\tilde{y}_{a}(L)\left(f_{1}, f_{2}\right)$ is in this case equal to $230.32 \mathrm{~nm}$. As such, the cultural noise is the most relevant source of noise to be considered. Therefore, the final model for the controller synthesis is that given in (13). The controller will be designed taking into account frequential properties of the cultural noise in order to reduce r.m.s. $s_{\tilde{y}_{a}(L)}$ to less than $80 \mathrm{~nm}$ in the frequency range $\left[\begin{array}{ll}1 \mathrm{~Hz} & 1 \mathrm{kHz}\end{array}\right]$. 


\section{LPV MODELING}

\subsection{Polynomial LPV model}

To design a gain scheduled controller based on LMI and the $\mathrm{H} \infty$ theory (see (Apkarian et al. [1995]; Chilali et al. [1996]; Gahinet et al. [1996]; Scherer et al. [1997]; de Souza et al. [2005])) a LPV model is derived from the nonlinear model (13). This nonlinear model can be reformulated into an affine (i.e. linear) LPV model if the stiffness $k_{a}$ and the damping $d_{a}$ (see equations (8) and (10)) are selected as independent varying parameters for which only the lower and the upper bounds of variation are considered. In this case, well known gain scheduled control strategies based on the LPV $/ \mathrm{H} \infty$ methodology can be used (see an example in (Poussot-Vassal et al. [2008])).

In the case of comb drive actuators, the nonlinear parameters $k_{a}$ and $d_{a}$ are matched and they have a polynomial structure. Such properties are not taken into account with a classical affine $\mathrm{LPV} / \mathrm{H} \infty$ design which is very conservative. In the present work, the use of the operating point $\delta_{a}$ as the only varying parameter is proposed. Using a Jacobian linearization, the nonlinear plant (13) is formulated into a polynomial LPV model of the form

$$
\begin{gathered}
G_{a}\left(s, \delta_{a}\right):\left\{\begin{array}{l}
\dot{X}_{p}(t)=A_{p}\left(\delta_{a}\right) X_{p}(t)+B_{p}\left[\begin{array}{l}
U(t) \\
b(t)
\end{array}\right] \\
y_{a}(L, t)=C_{p} X_{p}(t)
\end{array}\right. \\
A_{p}\left(\delta_{a}\right)=\left[\begin{array}{cc}
0 & 1 \\
\frac{-k_{1 a}}{m_{a}} & \frac{-d_{0 a}}{m_{a}}
\end{array}\right]+\delta_{a}\left[\begin{array}{cc}
0 & 1 \\
\frac{-2 k_{2 a}}{m_{a}} & \frac{-d_{1 a}}{m_{a}}
\end{array}\right]+\cdots \\
\cdots+\delta_{a}^{2}\left[\begin{array}{cc}
0 & 1 \\
\frac{-3 k_{3 a}}{m_{a}} & \frac{-d_{2 a}}{m_{a}}
\end{array}\right]+\delta_{a}^{3}\left[\begin{array}{cc}
0 & 1 \\
\frac{-4 k_{4 a}}{m_{a}} & \frac{-d_{3 a}}{m_{a}}
\end{array}\right]+\cdots \\
\cdots+\delta_{a}^{4}\left[\begin{array}{cc}
0 & 1 \\
\frac{-5 k_{5 a}}{m_{a}} & \frac{-d_{4 a}}{m_{a}}
\end{array}\right]+\delta_{a}^{5}\left[\begin{array}{cc}
0 & 1 \\
\frac{-6 k_{6 a}}{m_{a}} & 0
\end{array}\right] \\
B_{p}=\left[\begin{array}{cc}
0 & 0 \\
\frac{K_{e}}{m_{a} D_{a}} & \frac{K_{b t}}{m_{a}}
\end{array}\right], C_{p}=\left[\begin{array}{l}
1 \\
0
\end{array}\right]^{T}, X_{p}=\left[\begin{array}{l}
y_{a}(L) \\
\dot{y}_{a}(L)
\end{array}\right]
\end{gathered}
$$

where $A_{p} \in \mathbb{R}^{n_{a} \times n_{a}}, B_{p} \in \mathbb{R}^{n_{a} \times m}$ and $C_{p} \in \mathbb{R}^{p \times n_{a}}$, with $n_{a}=2, m=2$ and $p=1$.

To simplify notations, from now on, the variable $\tilde{y}_{a}(L)$ will be designed by $y_{a}(L)$. The non-linearity arising from the square voltage is overcome by considering $U=V_{i n}^{2}$ as the input of the model for the controller design. This consideration is performed since only positive values of the input voltage are applied to the actuator.

By referring to Fig. 5, the amplitude of the 2nd eigenmode is at least 18 times smaller than that of the first eigenmode. It frequency is two times higher than that of the first eigenmode. Therefore, we assume that unmodeled dynamics due to the linearization have a very little influence on the closed loop dynamic of the nonlinear system.

In LMI based $\mathrm{H} \infty$ theory, controllers are derived form decision variables (i.e. solutions) of a set of LMI conditions depending on the varying parameters. Since varying parameters have an infinite number of values between their lower and upper bounds, deriving a LPV controller would require solving an infinite number of LMIs. To deal with this constraint, a commonly used solution consists in solving LMIs only on the vertices of a polytopic model. This solution can be easily applied when the LPV model of the system is affine w.r.t the varying parameters (see examples in (Biannicet al. [1996];Poussot-Vassal et al. [2008])).

For model (20), the varying parameter enters in a polynomial way into the system definition which causes some issues for the controller derivation. To deal with this problem, a Linear Fractional Transformation of the model (20) is proposed. The polynomial LPV model is translated into an affine LPV descriptor model using a singular implicit modeling by a LFT. Afterwards, a polytopic controller is derived considering control specifications and specific LMI conditions (Fig.9).

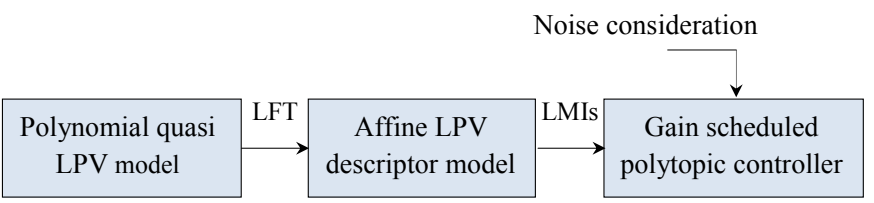

Fig. 9. Derivation of the gain scheduled polytopic controller from the polynomial LPV model.

\subsection{Derivation of an affine LPV descriptor model}

In order to simplify the parametric dependence of the state matrix $A_{p}\left(\delta_{a}\right)$ and to define an affine LPV descriptor model, a LFT (see Fig.10) is performed from the model $G_{a}\left(s, \delta_{a}\right)$ such that:

$$
\left[\begin{array}{c}
\dot{X}_{p} \\
y_{a} \\
z_{p}
\end{array}\right]=\left[\begin{array}{lll}
N_{11} & N_{12} & N_{13} \\
N_{21} & N_{22} & N_{23} \\
N_{31} & N_{32} & N_{33}
\end{array}\right]\left[\begin{array}{c}
X_{p} \\
U_{b} \\
v_{p}
\end{array}\right]
$$

where $z_{p} \in \mathbb{R}^{n_{z}}$ and $v_{p} \in \mathbb{R}^{n_{v}}$ are the input and output signals of the uncertainty block (i.e. varying parameter $\delta_{a}$ ) as shown in Fig.10, with $v_{p}=\delta_{a} z_{p}$.

The sub-matrices $\left\{N_{i, j}\right\}_{i, j=1, \ldots, 3}$ are defined from the interconnection of the LFT. The sizes of the vectors $v_{p}$ and $z_{p}$ are related to the polynomial orders of the stiffness and the damping. As such, $n_{z}$ and $n_{v}$ are defined by $n_{z}=n_{v}=7$ (i.e. order of the highest polynomial +1 ).

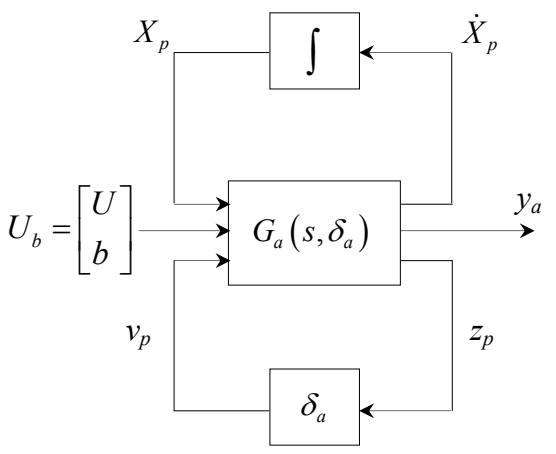

Fig. 10. Linear Fractional Transformation of the polynomial LPV model.

To transform the polynomial LPV model to an affine descriptor model, a new state vector $X_{\text {des }}=\left[\begin{array}{cc}X_{p}^{T} & v_{p}^{T}\end{array}\right]^{T}$ is chosen. The obtained affine LPV descriptor model is:

$G_{\text {des }}\left(s, \delta_{a}\right):\left\{\begin{array}{l}E \dot{X}_{\text {des }}(t)=A_{\text {des }}\left(\delta_{a}\right) X_{\text {des }}(t)+B_{\text {des }}\left[\begin{array}{l}U(t) \\ b(t)\end{array}\right] \\ y_{a}(L, t)=C_{\text {des }} X_{\text {des }}(t)\end{array}\right.$ 


$$
\begin{aligned}
& A_{\text {des }}=\left[\begin{array}{cc}
N_{11} & N_{13} \\
\delta_{a} N_{31} & \delta_{a} N_{33}+I_{n_{v}}
\end{array}\right], B_{\text {des }}=\left[\begin{array}{cc}
N_{12} & N_{12}^{\prime} \\
\delta_{a} N_{32} & \delta_{a} N_{32}
\end{array}\right] \\
& C_{\text {des }}=\left[\begin{array}{ll}
C_{p} & 0_{1 \times n_{v}}
\end{array}\right], \quad E=\operatorname{diag}\left(I_{n_{a}}, 0_{n_{v}}\right)
\end{aligned}
$$

$E$ is a singular matrix (i.e. non invertible matrix).

Moreover:

$$
\begin{aligned}
& N_{11}=\left[\begin{array}{cc}
0 & 1 \\
\frac{-k_{1 a}}{m_{a}} & \frac{-d_{0 a}}{m_{a}}
\end{array}\right]+\delta_{a}\left[\begin{array}{cc}
0 & 1 \\
\frac{-2 k_{2 a}}{m_{a}} & \frac{-d_{1 a}}{m_{a}}
\end{array}\right], N_{12}=\left[\begin{array}{l}
0 \\
\frac{K_{e}}{D_{a} m_{a}}
\end{array}\right] \text {, } \\
& N_{12}^{\prime}=\left[\begin{array}{l}
0 \\
\frac{k_{a}\left(\delta_{a}\right)}{m_{a}}
\end{array}\right] \\
& N_{13}=\delta_{a}\left[\begin{array}{ccccccc}
0 & 0 & 0 & 0 & 0 & 0 & 0 \\
\frac{-3 k_{3 a}}{m_{a}} & \frac{-4 k_{4 a}}{m_{a}} & \frac{-5 k_{5 a}}{m_{a}} & \frac{-6 k_{6 a}}{m_{a}} & \frac{-d_{2 a}}{m_{a}} & \frac{-d_{3 a}}{m_{a}} & \frac{-d_{4 a}}{m_{a}}
\end{array}\right] \text {, } \\
& N_{21}=\left[\begin{array}{ll}
1 & 0
\end{array}\right], N_{22}=0, N_{23}=0_{1 \times n_{v}},
\end{aligned}
$$

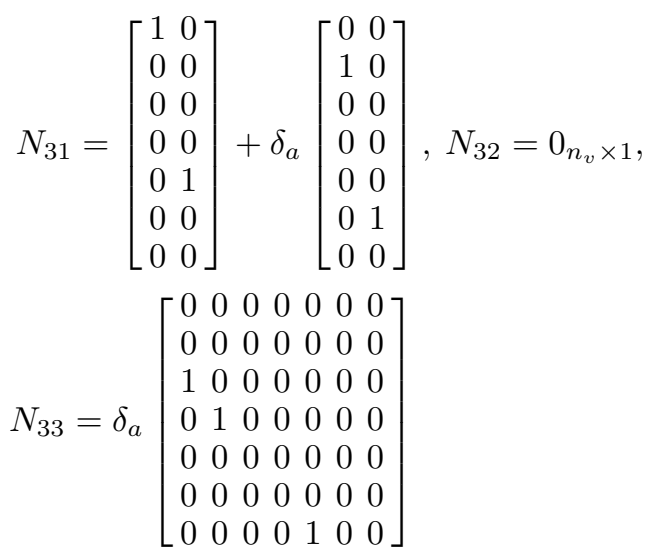

The vector $N_{12}^{\prime}$ describes the transfer between the cultural noise $b(t)$ and the state vector $X_{p}$. The PSD of the cultural noise is predominant at low frequencies (see Fig.6). As such, the effect of this source of noise on the positioning resolution is the most significant in the linear range of the actuation mechanism. Therefore, for the controller synthesis, $N_{12}^{\prime}$ is defined considering a frozen value of $\delta_{a}$ which is equal to $5 \mu \mathrm{m}$.

The descriptor model is used only for the synthesis of the scheduled controller via the resolution of a finite number of LMIs conditions. The controller is thereafter applied on the real microgripper.

\section{LPV BASED ROBUST CONTROL DESIGN}

The controller is designed based on the multivariable descriptor model (22). In order to achieve a fast and accurate positioning of the actuated arm in a large operating range (from few hundred nanometers to several tens of micrometers) with a nanometer resolution, control performances are defined as follow:

(1) The closed loop response time of the system must be lower than $30 \mathrm{~ms}$ ( 5 times smaller than the response time of the open loop system).

(2) No overshoot is admitted. An overshoot of few micrometers can destroy an object to be manipulated.
(3) The maximum static error lower than $0.1 \%$.

(4) The r.m.s of $y_{a}(L)$ must be lower than $80 \mathrm{~nm}$ in the frequency range $\left[\begin{array}{ll}1 \mathrm{~Hz} & 1 \mathrm{kHz}\end{array}\right]$.

In order to satisfy control specifications, two weighting transfer functions $W_{1}$ and $W_{2}$ are introduced. The weight $W_{1}$ allows tracking performances (1), (2) and (3) by applying constraints to the sensitivity function of the closed loop system (see Fig.11). The function $W_{2}$ is used for the cultural noise rejection.

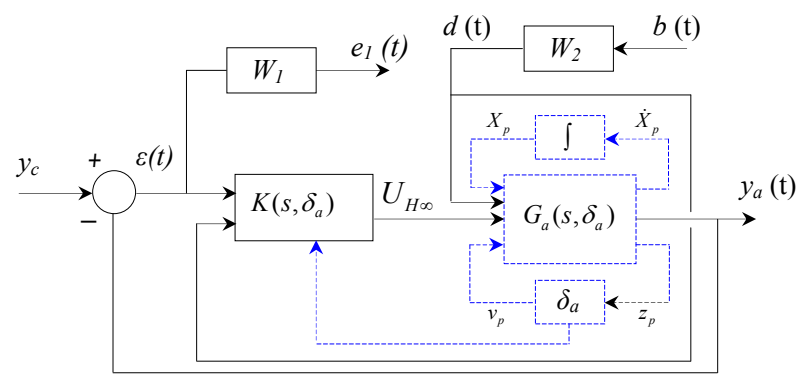

Fig. 11. Control scheme with the augmented LPV descriptor model considering the input noise $\mathrm{b}(\mathrm{t})$.

The controller is designed based on the multivariable descriptor model (22). The cultural noise can be measured in real time by fixing the laser spot of the interferometer on a solid block (see (Boudaoud et al. [2011]) for more details). The noise measurement is used by the controller to reduce the effect of $b(t)$ on the position $y_{a}(L)$ of the actuated arm. In this work, the use of a shaped form of the signal $b(t)$ as a second input of the controller for the synthesis is proposed (see Fig.11). This allows obtaining nanometer resolution while keeping a high bandwidth in closed loop.

\subsection{Control design}

The LPV control problem consists in finding a dynamic multivariable scheduled controller $K\left(s, \delta_{a}\right)$ that stabilize the closed loop system and minimize the $\mathrm{H} \infty$ norm $\gamma$ of a performance channel $\omega \rightarrow z$ for any value of the varying parameter $\delta_{a}$ between its lower bound $\delta_{a}$ and its upper

bound $\bar{\delta}_{a}$. Here, for the LPV design presented in Fig.11, the control problem is defined considering $\omega=\left[y_{c} b(t)\right]^{T}$ as the external input, $z=e_{1}$ as the controlled output, $U_{H \infty}$ as the control input and $\left[y_{a}(L, t) d(t)\right]^{T}$ as the measured variable.

From now on, let us consider the following transfer functions: $G y_{a / U_{H \infty}}=\frac{y_{a}(L)}{U_{H \infty}}, G y_{a / d}=\frac{y_{a}(L)}{d}, K_{U_{H \infty}}=\frac{U_{H \infty}}{\varepsilon}$ and $K_{U_{H \infty / d}}=\frac{U_{H \infty}}{d}$.

The relationship between the controlled output $z$ and the external input $\omega$ is defined by:

$$
\begin{aligned}
& e_{1}=\left(S \varepsilon_{/ y_{c}} W_{1}\right) y_{c} \\
& -\left(S \varepsilon_{y_{c}}\left(G y_{a / d}+G y_{a / U_{H \infty}} K_{U_{H \infty}}\right) W_{1} W_{2}\right) d
\end{aligned}
$$

where $S \varepsilon_{/ y_{c}}=\left[1+G y_{a / U_{H \infty}} K_{U_{H \infty}}\right]^{-1}$ is the sensitivity function.

Taking into account the relations (23), the control problem described above can be solved if the following 
conditions are satisfied for any admissible value of the varying parameter:

$$
\begin{aligned}
& \left\|S \varepsilon_{/ y_{c}}\right\|_{\infty} \leqslant \frac{\gamma}{\left\|W_{1}\right\|_{\infty}} \\
& \left\|S \varepsilon_{y_{c}}\left(G y_{a / d}+G y_{a / U_{H \infty}} K_{U_{H \infty} / \varepsilon}\right)\right\|_{\infty} \leqslant \frac{\gamma}{\left\|W_{1} W_{2}\right\|_{\infty}}
\end{aligned}
$$

To design $W_{1}$, a reference closed loop transfer function $T_{d}$ is defined based on control specifications:

$$
T_{d}=\frac{K_{d}}{\frac{1}{\omega_{d}^{2}} s^{2}+\frac{2 \xi_{d}}{\omega_{d}} s+1}
$$

where $K_{d}=0.9995, \omega_{d}=500 \mathrm{rad} / \mathrm{s}$ and $\xi_{d}=1.5 \mathrm{Ns} / \mathrm{m}$.

The weighting function $W_{1}$ is designed considering the desired closed loop dynamic behavior of the system. Hence:

$$
W_{1}=\frac{1}{1-T_{d}}
$$

For the noise rejection, the weight $W_{2}$ is selected such that the $\mathrm{H} \infty$ norm of the transfer $\varepsilon / d$ is reduced by $10 \mathrm{~dB}$ in the frequency range $\left[\begin{array}{ll}1 \mathrm{~Hz} & 10 \mathrm{~Hz}\end{array}\right]$ where the cultural noise is the most significant (see Fig.6) and by $6 \mathrm{~dB}$ in the frequency range $[10 \mathrm{~Hz} \quad 350 \mathrm{~Hz}$. This ensures a positioning resolution of less than $80 \mathrm{~nm}$ despite of the cultural noise. Thus, for nominal performances, $W_{2}$ is defined as:

$$
W_{2}=\frac{6.39 \times 10^{-8} s^{2}+8.75 \times 10^{-7} s+3}{1 \times 10^{-7} s^{2}+1 \times 10^{-7} s+1.5}
$$

Now, considering the LPV descriptor model (22) and the weight functions, a generalized augmented plant description is introduced:

$$
P_{\text {des }}\left(s, \delta_{a}\right):\left\{\begin{array}{l}
E_{g} \dot{X}_{g}(t)=A_{g}\left(\delta_{a}\right) X_{g}(t)+B_{1 g} \omega(t)+B_{2 g} U(t) \\
z(t)=C_{1 g} X_{g}(t)+D_{11 g} \omega(t)+D_{12 g} U(t) \\
{\left[\begin{array}{l}
y_{a}(L, t) \\
d(t)
\end{array}\right]=C_{2 g} X_{g}(t)+D_{21 g} \omega(t)}
\end{array}\right.
$$

where :

$A_{g} \in \mathbb{R}^{r \times r}, \quad B_{1 g} \in \mathbb{R}^{r \times n_{w}}, \quad B_{2 g} \in \mathbb{R}^{r \times 1}, \quad C_{1 g} \in \mathbb{R}^{n_{z} \times r}$, $C_{2 g} \in \mathbb{R}^{2 \times r}, D_{11 g} \in \mathbb{R}^{n_{z} \times n_{w}}, D_{12 g} \in \mathbb{R}^{n_{z} \times r}, D_{21 g} \in \mathbb{R}^{2 \times n_{w}}$, with $r=n_{a}+n_{v}+n_{w}$

The real $n_{w}$ is equal to the order of $W_{1}+$ the order of $W_{2}$. Moreover, $E_{g}=\operatorname{diag}\left[E, I_{n w}\right]$.

In order to derive the controller $K\left(s, \delta_{a}\right)$ satisfying required closed loop performances, it is necessary to use specific LMI conditions that takes into account that the matrix $E_{g}$ is singular. Among LMIs that meet this problem, one can use those proposed in (Masubuchi et al. [2004]) given as:

$$
\begin{aligned}
& {\left[\begin{array}{cc}
Y E_{g}^{T} & E_{g} \\
E_{g}^{T} & E_{g}^{T} \chi
\end{array}\right]=\left[\begin{array}{cc}
Y E_{g}^{T} & E_{g} \\
E_{g}^{T} & E_{g}^{T} \chi
\end{array}\right]^{T} \geqslant 0} \\
& {\left[\begin{array}{ccc}
M_{A}+M_{A}^{T} & M_{B} & M_{C}^{T} \\
M_{B}^{T} & -\gamma I & M_{D}^{T} \\
M_{C} & M_{D} & -\gamma I
\end{array}\right]<0}
\end{aligned}
$$

where

$$
\begin{aligned}
M_{A} & =\left[\begin{array}{cc}
A_{g}\left(\delta_{a}\right) Y^{T}+B_{2 g} F^{T} & A_{g}\left(\delta_{a}\right) \\
H^{T} & \chi^{T} A_{g}\left(\delta_{a}\right)+G^{T} C_{2 g}
\end{array}\right], \\
M_{B} & =\left[\begin{array}{l}
B_{1 g} \\
\chi^{T} B_{1 g}+G^{T} D_{21 g}
\end{array}\right], \\
M_{c} & =\left[\begin{array}{ll}
C_{1 g} Y^{T}+D_{12 g} F^{T} & C_{1 g}
\end{array}\right] \text { et } M_{D}=D_{11 g}
\end{aligned}
$$

The matrices $\chi$ and $Y$ are Lyapunov matrices which are considered constant herein. Decision variables of LMIs (29) are the matrices $\chi, Y, F, G$ and $H$ with appropriate dimensions.

As emphasized in Section.4.1, the LMI problem (29) results in an infinite set of LMIs to solve since $\delta_{a} \in$ $\left[\begin{array}{ll}\delta_{a} & \bar{\delta}_{a}\end{array}\right]$. Nevertheless, thanks to the affine dependence of $A_{d e s}\left(\delta_{a}\right)$ w.r.t the varying parameter, the LMI can be solved only on the vertices (see (Zin et al. [2006]) for an example) of the set $\delta_{a} \in\left[\delta_{a} \bar{\delta}_{a}\right]$. Here, two sets of LMIs must be solved because one varying parameter is considered. If the LMI problem is feasible, the solution leads to a LPV polytopic controller with the following state space representation:

$$
K\left(s, \delta_{a}\right)=\alpha_{1}\left(\delta_{a}\right)\left[\begin{array}{cc}
A_{k 1} & B_{k 1} \\
C_{k 1} & 0
\end{array}\right]+\alpha_{2}\left(\delta_{a}\right)\left[\begin{array}{cc}
A_{k 2} & B_{k 2} \\
C_{k 2} & 0
\end{array}\right]
$$

The couple $\left\{\alpha_{1}\left(\delta_{a}\right), \alpha_{2}\left(\delta_{a}\right)\right\}$ is defined by:

$$
\alpha_{1}\left(\delta_{a}\right)=\frac{\bar{\delta}_{a}-\delta_{a}}{\delta_{a}-\delta_{a}} \text { et } \alpha_{2}\left(\delta_{a}\right)=1-\alpha_{1}\left(\delta_{a}\right)
$$

For the computation of the matrices $\left\{A_{k i}, B_{k i}, C_{k i}\right\}_{i=1,2}$, the generalized augmented descriptor plant (28) is translated back into a polytopic form. At each vertex of the polytope, the affine descriptor model must be translated into a polynomial form. To address this requirement, the following transformation is performed: for a vertex $i$, the affine LPV descriptor model that can be represented by the form

$$
\begin{aligned}
& P_{d e s_{i}}:\left[\begin{array}{ccc}
{\left[A_{g_{i}}\right]} & {\left[B_{1 g_{i}}\right]} & {\left[B_{2 g_{i}}\right]} \\
{\left[C_{1 g_{i}}\right]} & {\left[D_{11 g_{i}}\right]} & {\left[D_{12 g_{i}}\right]} \\
{\left[C_{2 g_{i}}\right]} & {\left[D_{21 g_{i}}\right]} & 0
\end{array}\right] \\
& =\left[\begin{array}{ccc}
{\left[\begin{array}{ll}
A_{11 g_{i}} & A_{12 g_{i}} \\
A_{21 g_{i}} & A_{22 g_{i}}
\end{array}\right]} & {\left[\begin{array}{c}
B_{11 g_{i}} \\
B_{12 g_{i}}
\end{array}\right]} & {\left[\begin{array}{c}
B_{21 g_{i}} \\
B_{22 g_{i}}
\end{array}\right]} \\
{\left[\begin{array}{ll}
C_{11 g_{i}} & C_{12 g_{i}}
\end{array}\right]} & {\left[D_{11 g_{i}}\right]} & {\left[D_{12 g_{i}}\right.}
\end{array}\right]
\end{aligned}
$$

is equivalent to the polynomial LPV model

$$
\begin{aligned}
& P_{\text {pol }_{i}}:\left[\begin{array}{ccc}
A_{s_{i}} & B_{1 s_{i}} & B_{2 s_{i}} \\
C_{1 s_{i}} & D_{11 s_{i}} & D_{12 s_{i}} \\
C_{2 s_{i}} & D_{21 s_{i}} & 0
\end{array}\right]=\left[\begin{array}{ccc}
A_{11 g_{i}} & B_{11 g_{i}} & B_{21 g_{i}} \\
C_{11 g_{i}} & D_{11 g_{i}} & D_{12 g_{i}} \\
C_{21 g_{i}} & D_{21 g_{i}}
\end{array}\right] \\
& -\left[\begin{array}{c}
A_{12 g_{i}} \\
C_{12 g_{i}} \\
C_{22 g_{i}}
\end{array}\right] A_{22 g_{i}}^{-1}\left[\begin{array}{lll}
A_{21 g_{i}} & B_{12 g_{i}} & B_{22 g_{i}}
\end{array}\right]
\end{aligned}
$$

This transformation is feasible if the following conditions are satisfied:

(1) $B_{12 g_{i}}=0$ and $C_{12 g_{i}}=0$,

(2) $B_{22 g_{i}}=0$ and $C_{22 g_{i}}=0$;

(3) $A_{22 g_{i}}$ is an invertible matrix. 
The conditions (2) and (3) are satisfied in this work. However, the first condition is not satisfied. Therefore, in the generalized state vector $X_{g}$ of (28), the state vector $X_{W 1}$ of the weight function $W_{1}$ and the state vector $X_{W 2}$ of the weight function $W_{2}$ are placed from the line $n_{a}+1$. In other words, initially $X_{g}$ was defined by

$$
X_{g}=\left[\begin{array}{l}
X_{d e s} \\
X_{W 1} \\
X_{W 2}
\end{array}\right]=\left[\begin{array}{l}
X_{p} \\
v_{p} \\
X_{W 1} \\
X_{W 2}
\end{array}\right]
$$

and now, it becomes

$$
X_{g}=\left[\begin{array}{l}
X_{p} \\
X_{W 1} \\
X_{W 2} \\
v_{p}
\end{array}\right]
$$

Finally, the matrices $\left\{A_{k i}, B_{k i}, C_{k i}\right\}_{i=1,2}$ are computed from the decision variables of the LMIs and from the matrices $\left\{A_{s i}, C_{2 s i}, B_{2 s i}\right\}_{i=1,2}$ of the polynomial model. The controller is implemented into a transfer function form.

\subsection{Control implementation}

In this section, we first compare the performance of two LTI controllers with the performance of the proposed gain scheduled controller in ideal conditions (low noise condition). This work shows the importance of using a scheduled controller in the case of the microgripper. Thereafter, the input noise $b(t)$ is taken into account for the design of the multivariable scheduled controller aiming at satisfying precision requirements.

\subsection{Low noise condition}

Control strategies are implemented considering that the microgripper is working on a vibration isolation table. Single input/output type controllers are sufficient in this case (the noise input is not considered). In addition to the gain scheduled controller, a LTI $/ \mathrm{H} \infty$ controller and a PID controller are designed for comparison.

For the scheduled controller, the control problem is solved using the LMIs (29). The gain $K_{b t}$ is set at zero and only the weight $W_{1}$ is taken into account in the augmented LPV descriptor model (see Fig.12). Here, the control problem is defined considering $\omega=y_{c}$ as the external input, $z=e_{1}$ as the controlled output, $U_{H \infty}$ as the control input and $y_{a}(L, t)$ as the measured variable (see Fig.12). The LMIs are then solved on each vertex of the polytopic model using the Yalmip interface (Lofberg et al. [2004]) with the solver SeDuMi (Sturm et al. [1999]). For the resolution, the bounds of $\delta_{a}$ have been defined such that $\delta_{a}=1 \mu \mathrm{m}$ and $\bar{\delta}_{a}=80 \mu \mathrm{m}$.

When considering this set, the optimal obtained performance indice $\gamma$ is equal to 2.73 ensuring a modulus margin greater than $\frac{1}{\gamma}=0.36$. Therefore, two $4^{\text {th }}$ order controllers $G_{k i}\left\{A_{k i}, B_{k i}, C_{k i}, 0\right\}_{i=1,2}$ are obtained.

The frequency responses of the sensitivity function $S \varepsilon_{/ y_{c}}=\frac{\varepsilon(t)}{y_{c}(t)}$ is computed and is compared with the frequency response of $\frac{1}{W_{1}}$. The computation is performed considering $5 \mu \mathrm{m}<\delta_{a}<80 \mu \mathrm{m}$. Results are pre-

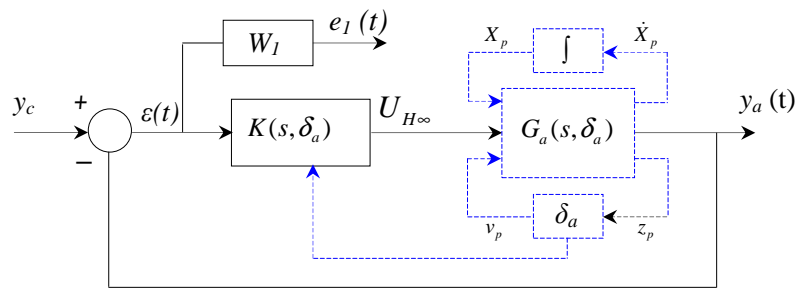

Fig. 12. Control scheme with the augmented LPV descriptor model considering the input noise $\mathrm{b}(\mathrm{t})=0$. Here $K_{b t}=0$ in $G_{a}\left(s, \delta_{a}\right)$.

sented in Fig.13. The obtained bandwidths are of about $500 \mathrm{rad} / \mathrm{s}$.

The LTI $/ \mathrm{H} \infty$ controller is designed using the polynomial LPV model (20) for a frozen value $\delta_{a}=5 \mu \mathrm{m}$ and for $K_{b t}=0$. The controller is based on the scheme presented in Fig.12 (without the scheduling part). In other words, the LTI $/ \mathrm{H} \infty$ control problem is defined considering also $\omega=y_{c}$ as the external input, $z=e_{1}$ as the controlled output, $U_{H \infty}$ as the control input and $y_{a}(L, t)$ as the measured variable. The same weighting function $W_{1}$ is used for the controller synthesis. As a result, a fourth order controller is obtained.

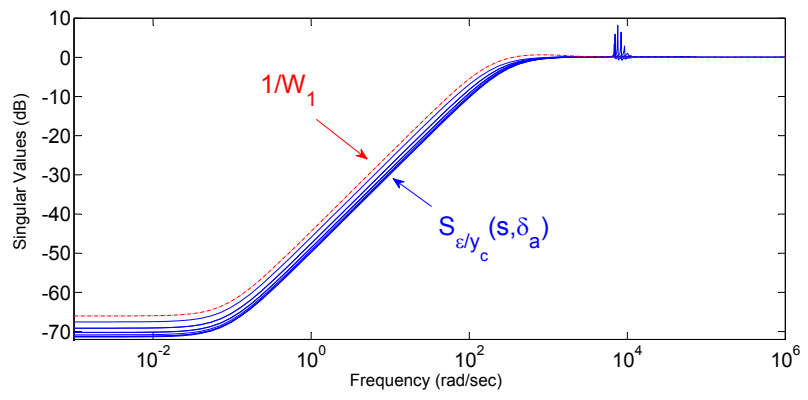

Fig. 13. Frequency responses of the sensitivity function (dynamic transfer $\varepsilon / y_{c}$ ) and that of the function $\frac{1}{W_{1}}$.

The PID controller is designed using the nonlinear model (11) and its parameters are identified using ZieglerNichols method. For an input reference $y_{c}=5 \mu \mathrm{m}$, the ultimate gain $K_{u}$ for which the output of the closed loop system oscillates with a constant amplitude is $K_{u}=8.91$. The period of such oscillations is $T_{u}=0.9 \mathrm{~ms}$. Therefore, the proportional, the derivative and the integral gains of the controller are respectively $K_{p}=5.34, K_{d}=1.12 \times 10^{-4}$ and $K_{i}=2.22 \times 10^{3}$. Real time implementation results of the three controllers are presented in section 6.2 .

\subsection{Significant noise condition}

In this condition, the microgripper is considered to be working out of a vibration isolation table. Therefore, we consider the multivariable control problem taking into account noise influence. The LMIs (29) are solved on each vertex of the polytopic model derived from (28). For the resolution, the bounds of $\delta_{a}$ have been defined such that $\delta_{a}=1 \mu \mathrm{m}$ and $\bar{\delta}_{a}=85 \mu \mathrm{m}$.

When considering this set, the optimal obtained performance indice $\gamma$ is equal to 5.65 ensuring a modulus margin greater than $\frac{1}{\gamma}=0.17$. Therefore, two $6^{\text {th }}$ order controllers $G_{k i}\left\{A_{k i}, B_{k i}, C_{k i}, 0\right\}_{i=1,2}$ are obtained. Each controller has two inputs $[\varepsilon(t) b(t)]$ and one output $U_{H \infty}$. In their transfer function form, the controllers are given in equations (33), (34), (35) and (36) (see also Fig.18). 


$$
\begin{gathered}
G_{k 11}=\frac{7.42 \times 10^{5} s^{5}+1.25 \times 10^{9} s^{4}+6.89 \times 10^{13} s^{3}+1.09 \times 10^{17} s^{2}+1.6 \times 10^{21} s+2.38 \times 10^{24}}{s^{6}+2519 s^{5}+4.65 \times 10^{9} s^{4}+7.64 \times 10^{12} s^{3}+2.13 \times 10^{17} s^{2}+3.19 \times 10^{20} s+2.66 \times 10^{19}} \\
G_{k 12}=\frac{7.95 \times 10^{5} s^{5}+4.33 \times 10^{9} s^{4}+2.44 \times 10^{14} s^{3}+5.27 \times 10^{17} s^{2}+9.6 \times 10^{21} s+1.4 \times 10^{25}}{s^{6}+4.26 \times 10^{5} s^{5}+5.89 \times 10^{9} s^{4}+2.82 \times 10^{13} s^{3}+2.68 \times 10^{17} s^{2}+3.57 \times 10^{20} s^{1}+2.97 \times 10^{19}} \\
G_{k 21}=\frac{-1.69 \times 10^{5} s^{5}-8.05 \times 10^{11} s^{4}-1.32 \times 10^{15} s^{3}-3.74 \times 10^{19} s^{2}-5.58 \times 10^{22} s-4.65 \times 10^{21}}{s^{6}+2519 s^{5}+4.65 \times 10^{9} s^{4}+7.64 \times 10^{12} s^{3}+2.13 \times 10^{17} s^{2}+3.19 \times 10^{20} s+2.66 \times 10^{19}} \\
G_{k 22}=\frac{7.39 \times 10^{5} s^{5}-9.86 \times 10^{11} s^{4}-4.87 \times 10^{15} s^{3}-4.62 \times 10^{19} s^{2}-6.13 \times 10^{22} s-5.11 \times 10^{21}}{s^{6}+4.26 \times 10^{5} s^{5}+5.89 \times 10^{9} s^{4}+2.82 \times 10^{13} s^{3}+2.68 \times 10^{17} s^{2}+3.57 \times 10^{20} s^{1}+2.97 \times 10^{19}}
\end{gathered}
$$

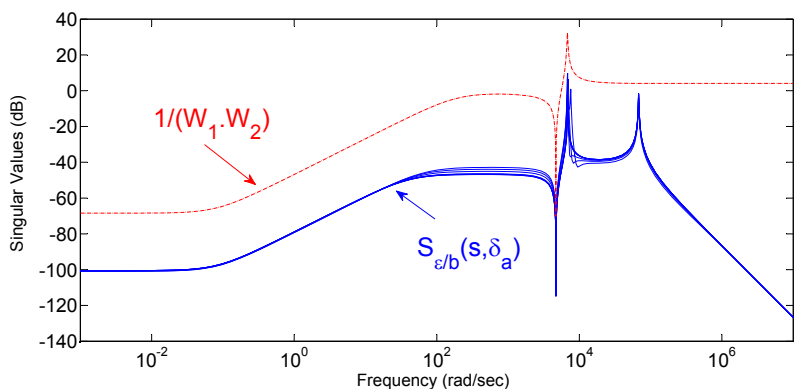

Fig. 14. Frequency responses of the dynamic transfer $\varepsilon / b$ and that of the function $\frac{1}{W_{1} W_{2}}$.

The frequency responses of the function $S \varepsilon_{/ b}=\frac{\varepsilon(t)}{b(t)}$ is computed and is compared with the frequency responses of $\frac{1}{W_{1} W_{2}}$. The computation is performed considering $100 \mathrm{~nm}<\delta_{a}<90 \mu \mathrm{m}$. Results are presented in Fig.14. Real time implementation results of the controller are presented in section 6.3 .

\section{EXPERIMENTAL SETUP AND REAL TIME IMPLEMENTATION}

\subsection{Experimental setup}

For the real time implementation of the controllers, the experimental setup of Fig.15 is used. It is composed of:

- The FT-G100 microgripper.

- A laser interferometer sensor (SP-120 SIOS Mebtechnik $\mathrm{GmbH}$ ) with two heads. The fist head (head 1) is used for the measurement of the actuated arm tip position. The second head (head 2) allows the real time measurement of the cultural noise.

- A 3 degrees of freedom (dof) positioning system which allows the alignment of the laser spot (head 1) with the gripping arm tip.

- A vibration isolation table.

- A controller board (dSPACE DS1103) with a Real Time Interface (RTI).

- An amplifier used to amplify the voltage signal at the output of the controller board which is limited to $10 \mathrm{~V}$.

Each controller is designed through the Matlab/Simulink software and is implemented into the controller board. The RTI allows applying different kind of reference trajectories $y_{c}$. The laser spot of the interferometer head 1 is fixed on the actuated arm tip. The laser spot of the interferometer head 2 is fixed on a solid block. Measured signals are taken into account in real time by the controller board through a

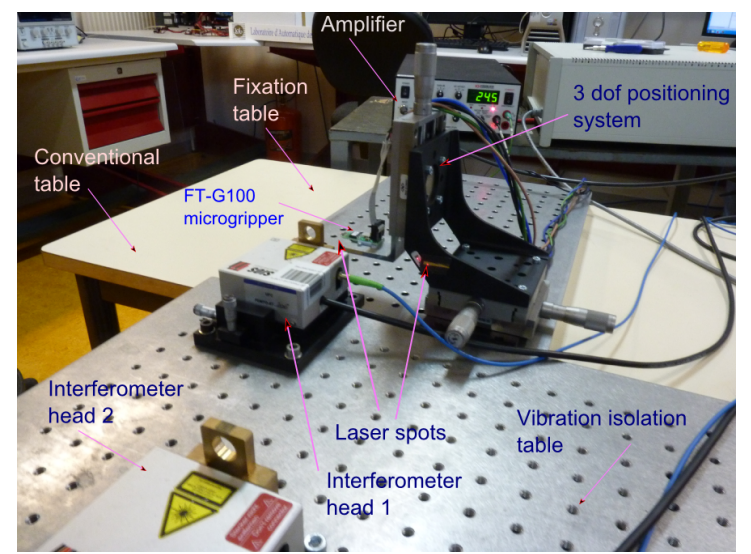

Fig. 15. Experimental setup when the microgripper is working out of the vibration isolation table.

digital/digital interface. The root square of the controller output (i.e. $\sqrt{U_{H \infty}}$ ) is applied to the comb drive actuator.

\subsection{Controllers implementation in low noise condition}

The controllers designed in section 5.3 are implemented in real time on the FT-G100 microgripper with $20 \mathrm{kHz}$ sampling frequency. In such condition, the 3 dof positioning system is fixed on the vibration isolation table. Only the head 1 of the interferometer is used for the measurement. The measured signal is used as a feedback signal.

The sub figures (a), (b) and (c) of Fig.16 show the normalized (to unity) controlled positions $y_{a}(L)$ for step references $y_{c}$ in the set $\left[\begin{array}{ll}5 \mu \mathrm{m} & 65 \mu \mathrm{m}\end{array}\right]$. Results are obtained with the scheduled controller Fig.16.a, the LTI/H $\infty$ controller Fig.16.b and the PID controller Fig.16.c. With the scheduled controller, the worst (i.e. highest) response time is $13.64 \mathrm{~ms}$, there is no overshoot and the highest static error is equal to $0.06 \%$. With the LTI $/ \mathrm{H} \infty$ controller, the worst response time is much bigger $(38.92 \mathrm{~ms})$, there is no overshoot and the highest static error is equal to $0.02 \%$. With the PID controller, the worst response time is $81.12 \mathrm{~ms}$.

It is clear that the scheduled controller is essential to satisfy robust performances over a large operating range of the system. In Fig.17, position of the gripping arm $y_{a}(L)$ in response to a reference trajectory are presented (experimental results). The proposed gain scheduled control strategy gives in this case satisfactory results.

In the next section, experimental controlled positioning results of the actuated arm are shown with the LPV controller taken into account the noise influence. Experiments are performed out of the vibration isolation table. 

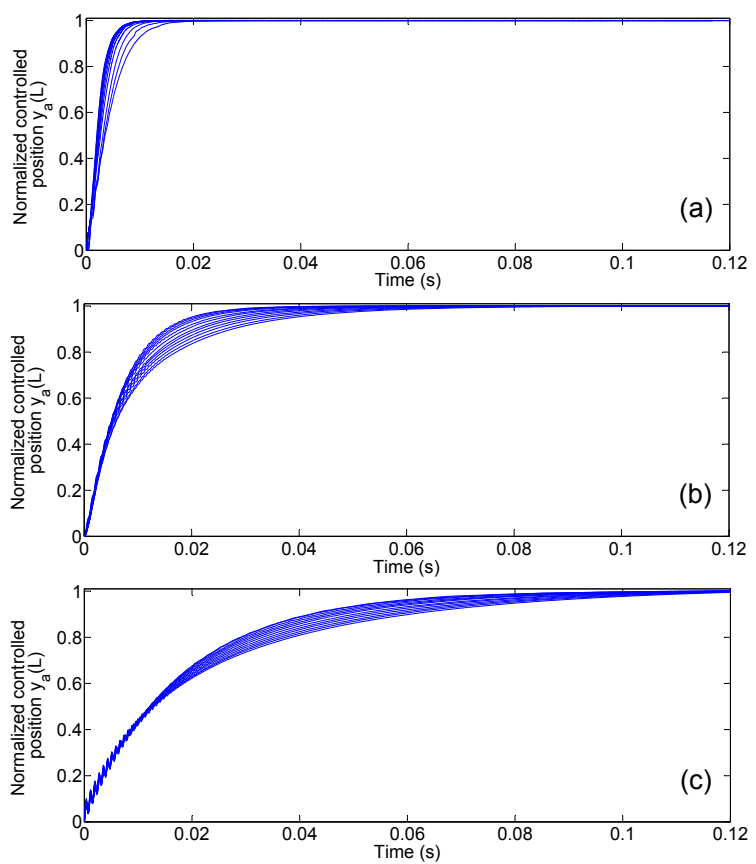

Fig. 16. Experimental normalized (to unity) step responses of the controlled gripping arm at different operating points (from $5 \mu \mathrm{m}$ to $65 \mu \mathrm{m}$ ) with the scheduled controller (a), the LTI/H $\infty$ controller (b) and with the PID controller (c).

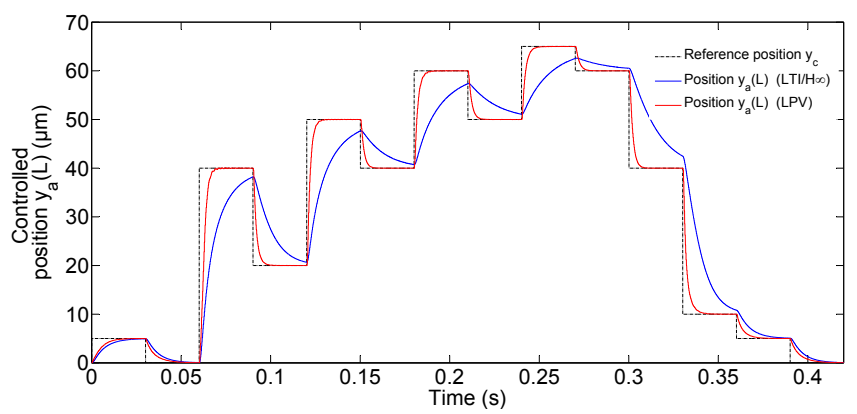

Fig. 17. Experimental position of the gripping arm $y_{a}(L)$ in response to a reference trajectory with the scheduled controller and with the LTI/H $\infty$ controller.

\subsection{Significant noise condition}

The scheduled controller designed in Section.5.4 is implemented on the microgripper which is located out of the vibration isolation table. The experimental setup is as shown in Fig.15. The position $y_{a}(L)$ is used as a feedback signal and also as scheduling variable for the controller (see Fig.18). The signal $b(t)$ is the experimental cultural noise measured in real time by the interferometer head 2 .

The multivariable controller faced real time implementation constraints. Since two digital/digital interfaces are used and since the controller is of high order (transfer functions of 6 th order), it was not possible to implement the controller with a sampling frequency higher than 5 $\mathrm{kHz}$. As such, the transfer functions (33), (34), (35) and (36) have been reduced into a third order. As such, the maximum sampling frequency that can be used is equal to $17 \mathrm{KHz}$.

Experiments show that the response time of the closed loop system is higher than the one measured with the single input single output LPV controller. However, for high amplitude displacements, the response time of the

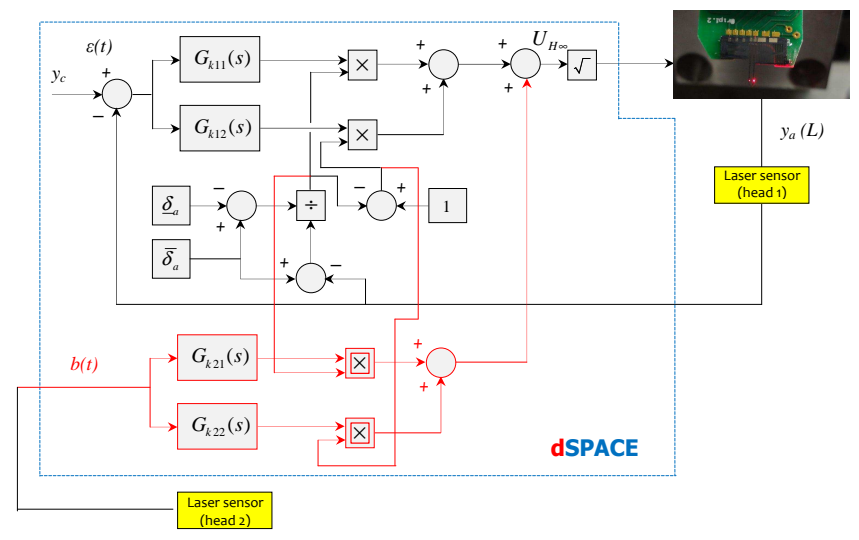

Fig. 18. Block diagram of the closed loop control system.

multivariable LPV controller remains smaller than of the LTI controllers.

To assess the effectiveness of the scheduled controller for small displacements, input references $y_{c}$ have been applied with amplitudes equal to $100 \mathrm{~nm}, 250 \mathrm{~nm}, 500 \mathrm{~nm}$, $750 \mathrm{~nm}, 1 \mu \mathrm{m}, 5 \mu \mathrm{m}, 10 \mu \mathrm{m}, 15 \mu \mathrm{m}$ and $20 \mu \mathrm{m}$. Results of the controlled displacements $y_{a}(L)$ are presented in Fig.19 and Fig.20. For each controller, experiments have been performed considering the case when the input $b(t)$ of the controller is taken into account (referred to "with noise rejection"), and the case when this input is not considered (i.e. the input $b(t)$ of the controller is set to zero) which is referred to "without noise rejection".

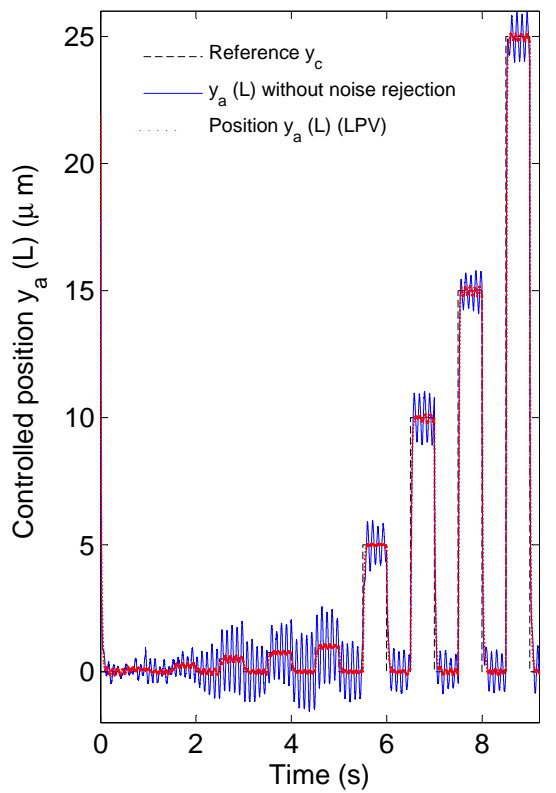

Fig. 19. Controlled positioning (experimental results) of the gripping arm for different input reference (100 $\mathrm{nm}, 250 \mathrm{~nm}, 500 \mathrm{~nm}, 750 \mathrm{~nm}, 1 \mu \mathrm{m}, 5 \mu \mathrm{m}, 10 \mu \mathrm{m}$, $15 \mu \mathrm{m}$ and $25 \mu \mathrm{m}$ ) with the multivariable scheduled controller.

Results show that it is crucial to consider the noise signal $b(t)$ when the system is subjected to important noise perturbation which is the case in robotics laboratory. This is fundamental for nanopositioning operations. When considering the noise input in the controller, some vibrations are undamped in the controlled position of the gripping arm. This is due to the measurement delay of the digital/digital interface. In Fig.21, nanopositioning simulation results are demonstrated using the multivariable LPV controller. The input noise is that measured experimentally in (Boudaoud et al. [2011]). In this case, since no delay is introduced, vi- 


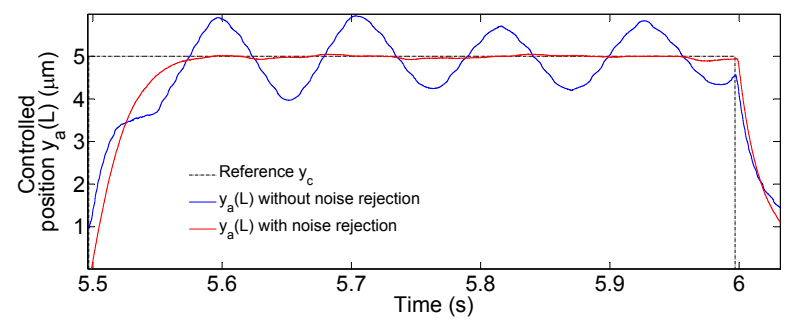

Fig. 20. Controlled positioning (experimental results) of the gripping arm for $5 \mu \mathrm{m}$ input reference with the multivariable scheduled controller.

brations are damped. Experimental results (with the delay of the measurements) show nevertheless very good results comparing with results obtained without considering the noise input.

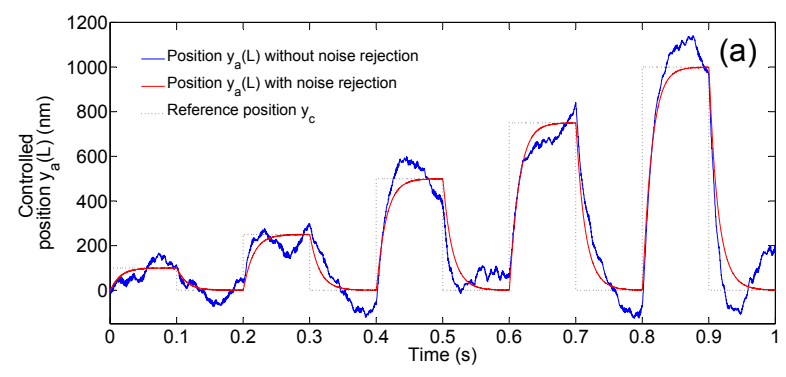

Fig. 21. Controlled positioning (simulation) of the gripping arm for different input reference $(100 \mathrm{~nm}, 250 \mathrm{~nm}$, $500 \mathrm{~nm}, 750 \mathrm{~nm}$ and $1 \mu \mathrm{m}$ ) with the multivariable scheduled controller.

\section{CONCLUSIONS}

In this paper, a new control approach of MEMS based electrostatic microgrippers is proposed. The controller allows positioning the gripping arm from few hundred nanometers to several tens of micrometers with a nanometer resolution and a fast response time despite of nonlinearities and significant noises. Such performances are greatly needed in biomedical microrobotics applications and have never been achieved previously with MEMS based microgrippers. To this end, a nonlinear dynamic model of the actuation mechanism has been proposed. First, it has been shown experimentally that the damping of the system is nonlinear and that the use of a third order polynomial to describe the nonlinear stiffness of a comb drive actuator is limited. Therefore, both the stiffness and the damping have been described by polynomials of higher orders. Afterward, two sources of noise that reduce the resolution of MEMS have been studied and the most relevant source of noise to be considered for the control design has been identified and introduced into the nonlinear model. Therefore, the nonlinear model has been reformulated into a polynomial LPV model. To deal with the particular structure of the LPV model, the later has been reformulated into an affine LPV descriptor model and a multivariable gain scheduled control strategy has been proposed. The effectiveness of the controller for noise rejection has been demonstrated experimentally and simulations showed that control results can be improved for nanopositioning if the measurement delay is canceled. The effectiveness of the controller for high amplitude displacements, in terms of precision and closed loop response time, has been demonstrated with experimental arguments. The control strategy allows new perspectives for high precision and high speed micromanipulation tasks in noisy laboratories environments.

\section{ACKNOWLEDGEMENTS}

This work has partially been supported by the French national project NanoRobust (ANR 2011 NANO 006) and by Hamecmopsys.

\section{REFERENCES}

Acar, C., Eler, S., PShkel, A. M. Concept, implementation, and control of wide bandwidth MEMS gyroscopes. American Control Conference, Arlington, USA , 2001.

Alwi, H., Zolotas, A., Edwards, C., Grigoriadis, K. Sliding Mode Control Design of an Electrostatic Microactuator using LPV schemes. American Control Conference, Montral, Canada, 2012.

Apkarian, P., Gahinet, P. A. Convex characterization of gain scheduled $\mathrm{H} \infty$ controllers. IEEE Transaction on Automatic Control, vol. 40, pp. 853-864, 1995.

Beyeler, F., Neild, A., Oberti, S., Bell, D., Yu, S., Bell, D., Dual, J., Nelson, B. J. Monolithically fabricated microgripper with integrated force sensor for manipulating microobjects and biological cells aligned in an ultrasonic field. Journal of microelectromechanical systems, vol. 16, pp. 7-15, 2007.

Biannic, J. M. Robust control of parameter varying systems: Aerospace applications. Ph.D. thesis, University Paul Sabatier, ONERA, 1996. (in French)

Borovic, B., Lewis, F.L., McCulley, W., QUN LIU, AI., Kolesar, E. S., POPA, D.O. Control Issues for Microelectromechanical Systems. IEEE Control Systems Journal, vol. 26, pp. 18-21, 2006.

Boudaoud, M., Haddab, Y., Le Gorrec, Y., Lutz, P. Noise characterization in millimeter sized micromanipulation systems. IFAC Mechatronics, vol. 21, pp. 1087-1097, 2011.

Boudaoud, M., Haddab, Y., Le Gorrec, Y., Lutz, P. Study of thermal and acoustic noise interferences in low stiffness AFM cantilevers and characterization of their dynamic properties. Review of Scientific Instruments, vol. 83, pp. 013704-, 2012.

Boudaoud, M., Haddab, Y., Le Gorrec, Y. Modeling and optimal force control of a nonlinear electrostatic microgripper. IEEE/ASME Transactions on mechatronics, vol. 18, pp. 1130-1139, 2013.

Bryzek, J., Abbott, H., Flannery, A., Cagle, D., Maitan, J. Control Issues for MEMS. IEEE Conference on Decision and Control, Maui, Hawaii, USA, 2003.

Callen, H. B., Welton, T. A. Irreversibility and Generalized Noise. Physical Review, vol. 83, pp. 3440, 1951.

Chaillet, N., Rgnier, S. Microrobotics for Micromanipulation. John Wiley and Sons Inc, ISBN: 978-1-84821-186$5,2010$.

Chilali, M., Gahinet, P. H $\infty$ design with pole placement constraints: An LMI approach. IEEE Transaction on Automatic Control, vol. 41, pp. 358-367, 1996.

de Souza, C. E., Trofino, A. Gain scheduled $\mathrm{H}_{2}$ controller synthesis for linear parameter varying systems via parameter-dependent lyapunov functions. International Journal of Robust and Nonlinear Control, vol. 16, pp. 243-257, 2005.

Ebrahimi, B., Bahrami, M. Robust sliding-mode control of a MEMS optical switch. Journal of Physics, vol. 34, pp. 728733, 2006.

Fei, J., Batur, C. Adaptive sliding mode control with sliding mode observer design for a MEMS vibratory gyroscope. ASME International Mechanical Engineering Congress and Exposition, Seattle,Washington, USA, 2007. 
Fei, J., Chowdhury, F. Robust Adaptive Sliding Mode Controller for Triaxial Gyroscope. IEEE Conference on Decision and Control, Shanghai, China, 2009.

Gahinet, P., Apkarian, P., Chilali, M. Affine parameterdependent Lyapunov functions and real parametric uncertainty. IEEE Transaction on Automatic Control, vol. 41, pp. 436-442, 1996.

Haddab, Y., Uccheddu, B. Commande robuste d'une pince microfabrique actionnement lectrostatique. Conference Internationale Francophone d'Automatique, Bucarest, Romania, 2008. (in French)

Kimura, Y., Yanagimachi, R. Intracytoplasmic sperm injection in the mouse. Biol. Reprod, vol. 52, pp. 709$720,1995$.

Kovacic, I., Brennan, M. J. The Duffing Equation: Nonlinear Oscillators and their Behaviour. John wiley and sons editions, ISBN-10: 0470715499, ISBN-13: 9780470715499, 2011.

Lee, K. B., Pisano, A. P., Lin, L. Nonlinear behaviors of a comb drive actuator under electrically induced tensile and compressive stresses. Journal of Micromechanics and Microengineering, vol. 17, pp. 557, 2007.

Legtenberg, R., Groeneveld, A., Elwenspoek, M. Comb drive actuators for large displacements. Journal of Micromechanics and Microengineering, vol. 6, pp. 320329, 1996.

Lofberg J. YALMIP : A Toolbox for Modeling and Optimization in MATLAB. CACSD Conference, Taipei, Taiwan, 2004.

Maithripala, D. H. S., Berg, J. M., Dayawansa, W. P. Capacitive stabilization of an electrostatic actuator : output feedback viewpoint. American Control Conference, Denver, Colorado, USA, 2003.

Masubuchi, I., Kato, J., Saeki, M., Ohara, A. Gain scheduled controller design based on descriptor representation of LPV systems: Application to flight vehicle control. IEEE Conference on Decision and Control, Atlantis, Bahamas, 2004.

Moussa, W. A., Ahmed, H., Badawy, W., Moussa, M. Investigating the reliability of electrostatic comb-drive actuators used in micro fluidic and space systems using finite element analysis. In Can. J. Elect. Comput. eng, vol. 27, pp. 195-200, 2002.

Muntwyler, S., Kratochvil, B. E., Beyeler, F., Nelson, B. J. Monolithically Integrated Two-Axis Microtensile Tester for the Mechanical Characterization of Microscopic Samples. Journal of microelectromechanical systems, vol. 19, pp. 1223-1233, 2010.

Pang, C. K., Lu, Y., Lin, C., Chen, J., Zhu, H., Yang, J., Moua, J., Guo, G., Chen, B. M., Lee, T. H. Design, fabrication, sensor fusion, and control of a micro X-Y stage media platform for probe-based storage systems. Mechatronics, vol. 19, pp. 1158-1168, 2009.

Poussot-Vassal, C, Sename, O., Dugard, L., Gaspar, P., Szabo, Z., Bokor, J. A new semi-active suspension control strategy through LPV technique. Control Engineering Practice, vol. 16, pp. 15191534, 2008.

Salah, M. H., Alwidyan, K. M., Tatlicioglu, E., Dawson, D. M. Robust Backstepping Nonlinear Control for ParallelPlate Micro Electrostatic Actuators. IEEE Conference on Decision and Control, Atlanta, Georgia, USA, 2010.

Scherer, C., Gahinet, P., Chilali, M. Multiobjective output-feedback control via LMI optimization. IEEE Transaction on Automatic Control, vol. 42, pp. 896-911, 1997.

Shirazi, F. A., Mohammadpour Velni, J., Grigoriadis, K. M. An LPV Design Approach for Voltage Control of an Electrostatic MEMS Actuator. Journal of Microelec- tromechanical Systems, vol. 20, pp. 302-311, 2011.

Sturm, J.F. Using SeDuMi 1.02, a matlab toolbox for optimization over symmetric cones. Optimization Methods and Software, vol. 11, pp. 625-653, 1999.

Sun, Y., Nelson, B. J. Biological cell injection using an autonomous microrobotic system. Int. J. Robot. Res, vol. 21, pp. 861-868, 2002.

Vagia, M., Nikolakopoulos, G., Tzes, A. Design of a robust PID-control switching scheme for an electrostatic microactuator. Control Engineering Practice, vol. 16, pp. 1321- 1328, 2008.

Vijayasai, A. P., Sivakumar, G., Mulsow, M ., Lacouture, S., Holness, A., Dallas, T. E. Haptic controlled threeaxis MEMS gripper system. Rev. Sci. Instrum, vol. 81, pp. 105114-, 2010.

Yamahata, C., Takekawa, T., Ayano, K., Hosogi, M., Kumemura, M., Legrand, B., Collard, D., Hashiguchi, G., Fujita, H. Silicon Nanotweezers with Adjustable and Controllable Gap for the Manipulation and Characterization of DNA Molecules. International Conference on Microtechnologies in Medicine and Biology, Okinawa, Japan, 2006.

Ye, W., Mukherjee, S., MacDonald, N. C. Optimal Shape Design of an Electrostatic Comb Drive in Microelectromechanical Systems. Journal of Microelectromechanical Systems, vol. 7, pp. 16-26, 1998.

Younis, M. I. MEMS Linear and Nonlinear Statics and Dynamics. Springer, ISBN 978-1-4419-6020-7, 2011.

Zhu, G., Lvine, J., Praly, L. Improving the performance of an electrostatically actuated MEMS by nonlinear control : advances and comparison. IEEE conference on decision and control, Seville, Spain, 2005.

Zin, A., Sename, O., Gaspar, P., Dugard, L., Bokor, J. An LPV/Hinf Active Suspension Control for Global Chassis Technology: Design and Performance Analysis. American Control Conference, Minneapolis, USA, 2006.- . 